\title{
A Robust Multi-Model Predictive Controller for Distributed Parameter Systems
}

\author{
Míriam R. García ${ }^{\mathrm{a}, \mathrm{b}}$, Carlos Vilas ${ }^{\mathrm{b}}$, Lino O. Santos ${ }^{\mathrm{c}}$, Antonio A. Alonso ${ }^{\mathrm{b}, *}$ \\ ${ }^{a}$ Hamilton Institute - National University of Ireland, Maynooth, Ireland \\ ${ }^{b}$ Process Engineering Group, IIM-CSIC, Eduardo Cabello 6, 36208 Vigo, Spain \\ ${ }^{c} C I E P Q P F$, Department of Chemical Engineering, \\ Faculty of Sciences and Technology, University of Coimbra, R. Silvio Lima, 3030-790
}

Coimbra, Portugal

\section{Abstract}

In this work a robust nonlinear model predictive controller for nonlinear convection-diffusion-reaction systems is presented. The controller makes use of a collection of reduced order approximations of the plant (models) reconstructed on-line by projection methods on POD (Proper Orthogonal Decomposition) basis functions. The model selection and model update step is based on a sufficient condition that determines the maximum allowable process-model mismatch to guarantee stable control performance despite process uncertainty and disturbances. Proofs on the existence of a sequence of feasible approximations and control stability are given.

Since plant approximations are built on-line based on actual measurements the proposed controller can be interpreted as a multi-model nonlinear predictive control (MMPC). The performance of the MMPC strategy is

\footnotetext{
${ }^{*}$ Corresponding author

Email addresses: Miriam.Garcia@nuim.ie (Míriam R. García), carlosvf@iim.csic.es (Carlos Vilas), lino@eq.uc.pt (Lino O. Santos), antonio@iim.csic.es (Antonio A. Alonso)
} 
illustrated by simulation experiments on a problem that involves reactant concentration control of a tubular reactor with recycle.

Keywords: Plant Model Mismatch; Proper Orthogonal Decomposition;

Controller Stability; Projection methods.

\section{Introduction}

The success of Model Predictive Control (MPC) industrial applications in the 1970s [1, 2] led to a wide spread of this control technology in industry [3]. The MPC algorithm relies on two main concepts: a predictive horizon to perform process dynamics model based predictions, and the solution of an open-loop control problem at every sampling-time. MPC proved over the years to be an efficient control tool for a wide class of multivariable nonlinear dynamic systems. Its appeal must be found essentially in its conceptual simplicity although, behind this formulation, a number of "not that simple" questions and problems hide. The vast and rich literature, with several books, e.g., $[4,5]$, available on this subject testifies the research efforts undertaken so far. It also reflects the degree of success attained in overcoming theoretical and practical issues related with stability, robustness and implementation. An early summary on predictive control research and applications can be found in [6]. Surveys on MPC theory and practice are given for instance in $[7,8]$, while $[9,10]$ provide an overview on robust MPC. The developments and challenges in nonlinear model predictive control (NMPC) are discussed in $[11,12,13,14,15]$. Optimality and stability issues related to MPC can be found in [16]. Robustness assessment of the controller has been recently addressed in the context of "Input to State Stability" concepts by [17, 18]. 
Distributed parameter systems (DPS) are examples of infinite dimensional nonlinear dynamic systems that challenge the efficiency of MPC implementations due to a number of reasons directly or indirectly related with the following issues: the high level of spatial discretization required to approximate the original set of partial differential equations; the dependence of the discretization scheme on the dynamic properties of the plant; the potentially stiff nature of the resulting nonlinear differential algebraic or ordinary equations or the usually large times of convergence associated with the subsequent optimization problems.

This may be tackled by formulating simpler ODE (Ordinary Differential Equation) models to describe the behavior of the DPS. For instance, in $[19,20]$ NMPC of a fixed-bed water-gas shift reactor is formulated using a simplified model with fewer equations and states. Also a lumped modeling approach is considered in [21] where a lexicographic optimization based MPC is applied to control a continuous pulp digester. In a strategy to apply on-line MPC to an experimental drying process [22], the partial differential equations are solved off-line, and then a linearized PDE (Partial Differential Equation) model around the previous off-line behavior is used to find the optimal variations for the on-line predictive control. This MPC strategy combines a two phase approximation of the PDE model in an internal model control (IMC) structure [23, 24]. Also, in [25, 26] to avoid the spatial discretization of the system the authors develop a neural network based model for the predictive controller. From another perspective, the development of more efficient NMPC algorithm strategies can greatly reduce the computational burden associated to the large-scale problems that arise in distributed 
parameter systems when using a full discretization of the PDE model [27].

Besides the various approaches taken in the aforementioned works, these challenges have been usually overcome in control and optimization applications by the so-called projection techniques [28, 29, 30, 31]. These techniques stand on the dissipative nature of the parabolic (diffusion based) PDE set and the time scale separation of dynamic modes, transforming the original PDE model into a low dimensional dynamic system capturing the most representative (slow) dynamics [32]. This approach has been successfully applied in the context of dynamic matrix control [33, 34] and MPC [35, 36, 37].

The so-called POD (Proper Orthogonal Decomposition) technique lies into this category. It was firstly proposed by Sirovich [38] and exploited by the group of Holmes and Lumley $[39,40,41]$ in the context of fluid dynamics as a way to explore the routes to turbulence phenomena, and was rapidly extended to other fields such as chemical reaction and biological systems. In this technique, measurements of the spatio-temporal evolution of the field are employed to derive the ROM (Reduced Order Model). We can envisage the application of these techniques in processes with process tomography devices to measure spatial state fields, that is, to capture the so called snapshots of the spatial distribution in order to track the states spatial variations inside the process. In fact, a recent review on control of DPS recognizes that the POD has emerged as a popular tool for model reduction towards modelbased control applications [42] and it has been exploited as an off-line model reduction technique for MPC [43].

The main disadvantage of PODs however, lies in that their accuracy is limited to a certain region of the state space defined by the field measure- 
ments employed to derive the ROM [44]. In the context of Model Predictive Control, this limitation translates into a divergence between the plant and its reduced order representation known as plant-process mismatch that if not properly assessed, for instance at the control design stage, it will deteriorate control performance or even drive the plant unstable. A detailed discussion about different approaches to waive this limitation can be found in [45] and references therein. Among those, particularly interesting is the work by [46] which presented a methodology to update the snapshots based on an optimization procedure which is able to find those regions where the ROM significantly differs from the full model. Although the aforementioned techniques increase the validity region of the POD technique, problems may arise when plant perturbations lead the system far away from such region. From another point of view, Atwell and King [47, 48] approached the problem for closed loop systems by using an alternative type of input collection which takes into account the derivation of the infinite dimensional feedback law. Using this input collection does not require guesswork and, as pointed out by the authors, controllers designed in this way perform better as compared with those designed using the traditional snapshot approach.

Independently of the order reduction technique, however, the dissipative nature of convection-diffusion-reaction systems ensures the existence of a reduced order representation capable of approximating at arbitrary accuracy the real plant dynamics for a given operation region [32]. Therefore, it is desirable to update the ROM currently in use by an MPC controller whenever closed loop performance and/or stability can not be guaranteed.

In this work a sufficient condition for robust stability of nominal MPC 
with modeling error similar to the one proposed in $[49,50]$ will be extended to the application of MPC of dissipative distributed process systems. It will be employed to select a reduced order model of the plant among a sequence of possible approximations so that the stabilizing properties of MPC are preserved despite uncertainties and disturbances. The authors in $[49,50]$ address the question of how much mismatch a given MPC algorithm can tolerate and how it can be assessed for stability of the MPC controller. Then an off-line procedure is developed to evaluate constants which determine sufficient conditions for robust stability. In this work, the proposed sufficient condition is applied to trigger the needed on-line updates of the ROM model used by the MPC controller in order to ensure robust stability. Therefore, when the sufficient condition is not satisfied, new plant measurements are taken to update on-line the POD basis, thus overcoming the reduced order model accuracy limitations discussed above.

Since the model is built on-line, based on actual measurements from the plant, the proposed approach can be interpreted as an MPC controller making use of multiple models, that is, a multi-model predictive control (MMPC). Early versions of the proposed strategy have been reported in $[51,52]$ and were applied to the control of simulated moving bed (SMB) separation processes [53]. The present contribution concentrates on the theoretical of the approach related with the model selection criterion and the convergence of the resulting control configuration.

The paper is organized as follows: The class of systems under consideration in this work is described in Section 2. This includes the role of dissipation in model reduction, the POD method and its main properties to be 
exploited later in the MMPC strategy. Section 3 summarizes the robust MPC approach to tackle plant/reduced order model mismatch and discusses conditions under which the closed-loop system remains stable. The final MMPC configuration comprising the reduced model update scheme and MPC, and its convergence properties, are described along Section 5. The performance of the MMPC strategy is illustrated by simulation experiments on a problem that involves the reactant concentration control of a tubular reactor with recycle (Section 6). Finally, main conclusions are drawn in Section 7.

\section{Dissipative systems and its dynamic approximations}

\subsection{System description and properties}

The class of systems we consider is that described by quasi-linear partial differential equations representing convection-diffusion-reaction mechanisms for mass or energy transport with (possibly non-linear) chemical reactions. For the sake of clarity we will concentrate in the present work on one dimensional partial differential equations. Note however that methods are not restricted to $1 \mathrm{D}$ but can be extended in a straightforward manner to $2 \mathrm{D}$ or $3 \mathrm{D}$ cases using arguments presented in $[32,54]$. Let the field $z(\xi, t)$ represent the evolution in space and time of a physical property, such as species concentration or temperature, in deviation form with respect to a given reference state. Formally, the resulting quasi-linear PDE takes the form:

$$
\frac{\partial z(\xi, t)}{\partial t}+\frac{\partial j(z)}{\partial \xi}=f(z)+p(\xi, t)
$$

where $\xi \in \mathbb{V}=[0, L] \subset \mathbb{R}$ denotes spatial position with $L$ being the length of the spatial domain and $t \in[0, \infty)$ denotes time. Function $j(z)$ includes both 
convective and diffusive fluxes so that:

$$
j(z)=v z(\xi, t)-k \frac{\partial z(\xi, t)}{\partial \xi} .
$$

As it is standard in tubular reactors, we will assume that the velocity field $v$ will be independent of space, thus $\frac{\partial v}{\partial \xi}=0$. Note however that equivalent conclusions can be drawn for the more general case of $v \equiv v(\xi)$ as it is described in [55]. The terms $f(z)$ and $p(\xi, t)$ represent the nonlinear reaction term and the, possibly distributed, control input, respectively. The system is completed with boundary conditions of the form:

$$
\left[n \cdot\left(k \frac{\partial z(\xi, t)}{\partial \xi}\right)+h z(\xi, t)\right]_{\xi=\mathbb{B}}=0,
$$

where $n$ is a unit normal vector pointing outwards of the boundary $\mathbb{B}$ and $h \geqslant$ 0 is a constant representing a property (mass or energy) transfer coefficient at the boundary. In what follows some of the function arguments will be omitted for the sake of clarity. We assume that the system is dissipative in the sense of references $[56,55]$. This implies, among other things, that $k$ in (2) must be positive and that the nonlinear term $f(z)$ satisfies the following assumption:

Assumption 1 (Lipschitz condition). There exist some nonnegative parameters $\mu$ and $\epsilon$ and a positive definite function $\ell(z, \mu) \geqslant 0$, with $\ell(z, 0)>0$ for $\|z\|>\epsilon$, such that the following relation holds for $f(z)$ in (1):

$$
z f(z)+\ell(z, \mu)=\mu z^{2} .
$$

Integrating (4) over the spatial domain $\mathbb{V}$ leads to

$$
\langle z, f\rangle_{\mathbb{V}}+L_{\mu}=\mu\langle z, z\rangle_{\mathbb{V}}, \quad L_{\mu}=\int_{\mathbb{V}} \ell(z, \mu) \mathrm{d} \xi,
$$


where $\langle g, h\rangle_{\mathbb{V}}$ should be understood as integration over $\mathbb{V}$, i.e., $\langle g, h\rangle_{\mathbb{V}}=$ $\int_{\mathbb{V}} g^{\mathrm{T}} h \mathrm{~d} \xi$. Given a field $z(\xi, t)$, let us define a $\mathcal{L}_{2}$ norm as follows: $\|z\|_{2}^{2}=$ $\langle z, z\rangle_{\mathbb{V}}$.

As shown in $[32,55]$ a field that obeys equations (1)-(4) is dissipative and therefore bounded in $\mathcal{L}_{2}$, provided that the control input field $p(\xi, t)$ is bounded. On the other hand, since $f(z)$ is Lipschitz, it is also bounded and thus belongs to $\mathcal{L}_{2}$. Because of this, both functions accept Fourier series expansions of the form [57]:

$$
z(t, \xi)=\sum_{i=1}^{\infty} m_{i}(t) \phi_{i}(\xi), \quad f(z)=\sum_{i=1}^{\infty} \sigma_{i}(t) \phi_{i}(\xi),
$$

where $m_{i}(t)$ and $\sigma_{i}(t)$ are the system dynamic modes, and $\phi_{i}(\xi)$ are eigenfunctions that constitute the solutions of the so-called Euler-Lagrange equation [58] (see also [32] for a discussion in the context of distributed passive systems):

$$
\frac{\partial^{2} \phi_{i}(\xi)}{\partial \xi^{2}}=-\lambda_{i} \phi_{i}(\xi)
$$

Equation (7) must be solved with the appropriate boundary conditions (in our case equation (3)) to produce a complete set of orthonormal eigenfunctions $\left\{\phi_{i}(\xi)\right\}_{i=1}^{\infty}$ with their corresponding positive eigenspectrum $\left\{\lambda_{i}\right\}_{i=1}^{\infty}$ where each $\lambda_{i}$ is an eigenvalue. As proved elsewhere (see for instance [58]), the eigenvalues are ordered such that $\lambda_{i} \leqslant \lambda_{j}$ for all integers $i, j$ whenever $i<j$ and $\lambda_{n} \rightarrow \infty$ as $n \rightarrow \infty$. To simplify the notation we denote $\phi_{i} \equiv \phi_{i}(\xi)$.

Note that projecting relation (7) over the eigenfunctions leads to:

$$
\left\langle\phi_{i}, \frac{\partial^{2} \phi_{j}}{\partial \xi^{2}}\right\rangle_{\mathbb{V}}=-\lambda_{j}\left\langle\phi_{i}, \phi_{j}\right\rangle_{\mathbb{V}}=-\lambda_{j} \delta_{i j},
$$

where $\delta_{i j}$ is the Kronecker delta. 
The background material we have just outlined in this section will allow us to construct a dynamic approximation of the plant capable of reproducing the behavior of the system at arbitrary precision (reduced order models). These will constitute the models employed by the predictive controllers to command the operation. A brief description of reduced order models (ROMs) is presented in Appendix A. The dynamics of the error associated to the approximation and its bounds are discussed next.

\subsection{Dynamic approximations}

Let us make use of relation (2) to rewrite system (1) as:

$$
\frac{\partial z(\xi, t)}{\partial t}+v \frac{\partial z(\xi, t)}{\partial \xi}=\frac{\partial}{\partial \xi}\left(k \frac{\partial z(\xi, t)}{\partial \xi}\right)+f(z)+p(\xi, t) .
$$

By what has been mentioned previously, fields in (9) accept expansion in Fourier series of the form of (6). Such series can be split into two contributions: one containing a finite number of elements $N$ and the other containing the remaining so that:

$$
\begin{array}{r}
z(\xi, t)=\sum_{i=1}^{\infty} m_{i}(t) \phi_{i}(\xi)=\sum_{i=1}^{N} m_{i}(t) \phi_{i}(\xi)+\sum_{i=N+1}^{\infty} m_{i}(t) \phi_{i}(\xi) \\
f(z)=\sum_{i=1}^{\infty} \sigma_{i}(t) \phi_{i}(\xi)=\sum_{i=1}^{N} \sigma_{i}(t) \phi_{i}(\xi)+\sum_{i=N+1}^{\infty} \sigma_{i}(t) \phi_{i}(\xi) \\
p(\xi, t)=\sum_{i=1}^{\infty} \pi_{i}(t) \phi_{i}(\xi)=\sum_{i=1}^{N} \pi_{i}(t) \phi_{i}(\xi)+\sum_{i=N+1}^{\infty} \pi_{i}(t) \phi_{i}(\xi) .
\end{array}
$$

In the same way first spatial derivatives of the field can be expressed by convergent series expansions since, as shown in Appendix B, they belong to $\mathcal{L}_{2}$ so that:

$$
\frac{\partial z(\xi, t)}{\partial \xi}=\sum_{i=1}^{\infty} \tau_{i}(t) \phi_{i}(\xi)=\sum_{i=1}^{N} \tau_{i}(t) \phi_{i}(\xi)+\sum_{i=N+1}^{\infty} \tau_{i}(t) \phi_{i}(\xi) .
$$


In the remaining of the paper, we will employ the subindex A to denote the part of the field related to the finite dimensional contribution in Eqns (10)(13) and a subindex B to refer to the part of the field corresponding to the infinite dimensional contribution. In this way,

$$
r_{\mathrm{A}}(\xi, t)=\sum_{i=1}^{N} \alpha_{i}(t) \phi_{i}(\xi) ; \quad r_{\mathrm{B}}(\xi, t)=\sum_{i=N+1}^{\infty} \alpha_{i}(t) \phi_{i}(\xi)
$$

with $r(\xi, t)$ representing any of the fields $z(\xi, t), f(\xi, t), p(\xi, t)$ or $\frac{\partial z(\xi, t)}{\partial \xi}$, and $\alpha(t)$ denoting the time dependent coefficients $m(t), \sigma(t), \pi(t)$ or $\tau(t)$.

In all the expressions above, $N+1$ will turn out to be the mode number after which one can assure that the remaining modes will relax exponentially fast. This will be shown next together with the fact that under Assumption 1 such $N$ can always be found.

To simplify the notation we denote $z \equiv z(\xi, t), z_{\mathrm{A}} \equiv z_{\mathrm{A}}(\xi, t), z_{\mathrm{B}} \equiv$ $z_{\mathrm{B}}(\xi, t)$. In addition, by orthogonality of the eigenfunctions and relations (10) we have that $\left\langle z_{\mathrm{A}}, z_{\mathrm{B}}\right\rangle_{\mathbb{V}}=0$, so that:

$$
\|z\|_{2}^{2}=\left\|z_{\mathrm{A}}\right\|_{2}^{2}+\left\|z_{\mathrm{B}}\right\|_{2}^{2}
$$

By the same token, and using expansions (10) and (13), it follows that:

$$
\left\langle z_{\mathrm{B}}, \frac{\partial z}{\partial t}\right\rangle_{\mathbb{V}}=\left\langle z_{\mathrm{B}}, \frac{\partial z_{\mathrm{B}}}{\partial t}\right\rangle_{\mathbb{V}} \text { and }\left\langle z_{\mathrm{B}}, \frac{\partial z}{\partial \xi}\right\rangle_{\mathbb{V}}=\left\langle z_{\mathrm{B}}, \frac{\partial z_{\mathrm{B}}}{\partial \xi}\right\rangle_{\mathbb{V}} .
$$

With these preliminary statements, let us define a Lyapunov function as $W=\frac{1}{2}\left\|z_{\mathrm{B}}\right\|_{2}^{2}$ and compute its time derivative along (9):

$$
\begin{aligned}
\frac{\mathrm{d} W}{\mathrm{~d} t}=\left\langle z_{\mathrm{B}}, \frac{\partial z_{\mathrm{B}}}{\partial t}\right\rangle_{\mathbb{V}}=-v\left\langle z_{\mathrm{B}}, \frac{\partial z_{\mathrm{B}}}{\partial \xi}\right\rangle_{\mathbb{V}} & +\left\langle z_{\mathrm{B}}, \frac{\partial}{\partial \xi}\left(k \frac{\partial z_{\mathrm{B}}}{\partial \xi}\right)\right\rangle_{\mathbb{V}} \\
& +\left\langle z_{\mathrm{B}}, f_{\mathrm{B}}\right\rangle_{\mathbb{V}}+\left\langle z_{\mathrm{B}}, p_{\mathrm{B}}\right\rangle_{\mathbb{V}} .
\end{aligned}
$$


Let us now examine the different terms in the RHS of equation (15). Using the first Green's identity, the convection term in (15) is given by:

$$
v\left\langle z_{\mathrm{B}}, \frac{\partial z_{\mathrm{B}}}{\partial \xi}\right\rangle_{\mathbb{V}}=\frac{1}{2} v \int_{\mathbb{V}} \frac{\partial}{\partial \xi}\left(z_{\mathrm{B}}^{2}\right) \mathrm{d} \xi=\frac{1}{2} v\left(z_{\mathrm{B}}^{2}(L)-z_{\mathrm{B}}^{2}(0)\right)
$$

or in terms of the Lyapunov function

$$
v\left\langle z_{\mathrm{B}}, \frac{\partial z_{\mathrm{B}}}{\partial \xi}\right\rangle_{\mathbb{V}}=v(W(L)-W(0))
$$

By assuming no disturbance at $\xi=0, z_{\mathrm{B}}^{2}(0)=2 W(0)=0$ which makes the left hand side of (17) positive definite.

Expanding the field of the diffusion term in (15) in series of the form (10) we have:

$$
\left\langle z_{\mathrm{B}}, \frac{\partial}{\partial \xi}\left(k \frac{\partial z_{\mathrm{B}}}{\partial \xi}\right)\right\rangle_{\mathbb{V}}=\left\langle\sum_{i=N+1}^{\infty} m_{i} \phi_{i}, k \sum_{i=N+1}^{\infty} m_{i} \frac{\partial^{2} \phi_{i}}{\partial \xi^{2}}\right\rangle_{\mathbb{V}} .
$$

Using (7) and orthonormality of eigenfunctions we get:

$$
\left\langle z_{\mathrm{B}}, \frac{\partial}{\partial \xi}\left(k \frac{\partial z_{\mathrm{B}}}{\partial \xi}\right)\right\rangle_{\mathbb{V}}=\left\langle\sum_{i=N+1}^{\infty} m_{i} \phi_{i}, k \sum_{i=N+1}^{\infty} m_{i}\left(-\lambda_{i}\right) \phi_{i}\right\rangle_{\mathbb{V}}=k \sum_{i=N+1}^{\infty}\left(-\lambda_{i}\right) m_{i}^{2} .
$$

Taking into account that the smallest eigenvalue in the previous series is $\lambda_{N+1}$ and $\left\|z_{\mathrm{B}}\right\|_{2}^{2}=\sum_{i=N+1}^{\infty} m_{i}^{2}$, the diffusive terms can be bounded as

$$
\left\langle z_{\mathrm{B}}, \frac{\partial}{\partial \xi}\left(k \frac{\partial z_{\mathrm{B}}}{\partial \xi}\right)\right\rangle_{\mathbb{V}} \leqslant-k \lambda_{N+1}\left\|z_{\mathrm{B}}\right\|_{2}^{2}=-2 k \lambda_{N+1} W
$$

Finally, invoking the Lipschitz condition (4) in its integral form (5), we derive a bound on the nonlinear term in (15), being of the form:

$$
\left\langle z_{\mathrm{B}}, f_{\mathrm{B}}\right\rangle_{\mathbb{V}} \leqslant-L_{\mu}+2 \mu W \leqslant 2 \mu W
$$


Combining (17), (18), and (19), with (15), we obtain the following inequalities:

$$
\begin{aligned}
\frac{\mathrm{d} W}{\mathrm{~d} t} & \leqslant-v W(L)-2 k \lambda_{N+1} W+2 \mu W+\left\langle z_{\mathrm{B}}, p_{\mathrm{B}}\right\rangle_{\mathbb{V}} \\
& \leqslant-2 \varepsilon_{N} W+\left\langle z_{\mathrm{B}}, p_{\mathrm{B}}\right\rangle_{\mathbb{V}}
\end{aligned}
$$

where $\varepsilon_{N}=k \lambda_{N+1}-\mu$. Note that because of the ordering property of eigenvalues $\lambda_{i}$ mentioned in the previous section, for a given $\mu$ and $k$ it is always possible to find a number of modes large enough such that $\varepsilon_{N}>0$. In fact, let $N$ be such number. Following these preliminary developments we establish a bound on the error associated to the $N$-mode approximation of the field, that is, a bound on $\left\|z_{\mathrm{B}}\right\|_{2}^{2}$ quite connected to the notion of input to state stability [59]. This is formally stated in the following proposition:

Proposition 1. Let $N$ be such that $\varepsilon_{N}=k \lambda_{N+1}-\mu>0$ and the input control field be bounded as $\left\|p_{\mathrm{B}}\right\|_{2} \leqslant \beta \sqrt{2}$ over a time interval $T$ with $\beta \in \mathbb{R}^{+}$. Then the error associated to the approximation is bounded as follows:

$$
\left\|z_{\mathrm{B}}(T)\right\|_{2}^{2} \leqslant\left\|z_{\mathrm{B}}(0)\right\|_{2}^{2} \exp \left(-\varepsilon_{N} T\right)+\frac{2 \beta^{2}}{\varepsilon_{N}^{2}}\left(1-\exp \left(-\varepsilon_{N} T\right)\right) .
$$

Proof: First note that since the control field $p_{\mathrm{B}}$ is assumed to be bounded, using Hölder's inequality, we have that $\left\langle z_{\mathrm{B}}, p_{\mathrm{B}}\right\rangle_{\mathbb{V}} \leqslant \sqrt{2}\left\|z_{\mathrm{B}}\right\|_{2} \beta$. Substituting in (20) and using the fact that $\left\|z_{\mathrm{B}}\right\|_{2}=\sqrt{2} W^{1 / 2}$ we have that:

$$
\frac{\mathrm{d} W}{\mathrm{~d} t} \leqslant-2 \varepsilon_{N} W+2 \beta W^{1 / 2} .
$$

Because the second term on the RHS (square root of $W$ ) is a concave function, it is upper bounded by its supporting hyperplane [56], what allows us to write:

$$
W^{1 / 2} \leqslant W_{1}^{1 / 2}+\frac{1}{2 W_{1}^{1 / 2}}\left(W-W_{1}\right) .
$$


Let $W_{1}$ be of the form $W_{1}=\frac{1}{\omega^{2}}\left(\frac{\beta}{2 \varepsilon_{N}}\right)^{2}$, with $0<\omega<1$ an auxiliary parameter. Substituting $W_{1}$ in (23), inequality (22) can be rewritten as:

$$
\begin{aligned}
\frac{\mathrm{d} W}{\mathrm{~d} t} & \leqslant-2\left(\varepsilon_{N}-\frac{\beta}{2 W_{1}^{1 / 2}}\right) W+\beta W_{1}^{1 / 2} \\
& \leqslant-2 \varepsilon_{N}(1-\omega) W+\frac{\beta^{2}}{2 \omega \varepsilon_{N}} .
\end{aligned}
$$

Multiplying both sides of the above inequality by $\exp (r t)$, with $r=2 \varepsilon_{N}(1-$ $\omega)$, and integrating over time in the interval $(0, T)$ we obtain:

$$
W(T) \leqslant \exp (-r T) W(0)+\frac{\beta^{2}}{4 \varepsilon_{N}^{2} \omega(1-\omega)}(1-\exp (-r T)) .
$$

The second term on the right hand side attains a minimum for $\omega=1 / 2$ for which $r=\varepsilon_{N}$. Consequently, a minimum bound for the propagation of the approximation error $\left\|z_{\mathrm{B}}\right\|_{2}^{2}$ takes the form given by Eqn (21).

Note that the larger $N$ (and hence $\varepsilon_{N}$ ), the smaller the bound on the field $z_{\mathrm{B}}$. Thus the error associated to neglecting the field $z_{\mathrm{B}}$ can be made arbitrarily small by increasing the number of modes in the field $z_{\mathrm{A}}$.

\section{Model Predictive Control. Problem Statement}

As it is well known in process control, the essential idea behind any model predictive controller is that of finding at regular time intervals those control actions which minimize a given cost function indicative of the process performance. To that purpose a reliable dynamic description of the process to be controlled, usually in the form of algebraic and difference or differential equations (DAES) is needed together with appropriate sensors to determine the present state of the process. 
For the case considered in this work, and in order to construct the cost function to be minimized during operation we define the state and control variables in deviation form with respect to a reference which is chosen as the controller setpoint, i.e.,

$$
z=\hat{z}-z_{\mathrm{sp}}, \quad p=\hat{p}-p_{\text {ref }}
$$

where $z \in \mathbb{R}^{q}$ and $p \in \mathbb{R}^{r}$ are the vector fields, and $q$ and $r$ are the number of states and control variables, respectively. From (25) it follows that at the setpoint we have that $z=0$, and $p=0$.

- Cost function at time $t_{k}$

In the MPC problem formulation we consider a predictive horizon $M$ and a control horizon $M_{\mathrm{c}}$, with $M_{\mathrm{c}} \leqslant M$. Let $z_{k}$, and $p_{k}$ represent the deviation variables at every sampling time $t_{k}, k \geqslant 0$. Let us define a positive definite function $h(z, p): \mathbb{R}^{q} \times \mathbb{R}^{r} \rightarrow \mathbb{R}^{+}$, and denote by $h\left(z_{k}, p_{k}\right)$ the value of such function at time $t_{k}$. A possible candidate cost function, frequently employed in the MPC formulations, is the quadratic function:

$$
h\left(z_{k}, p_{k}\right)=\frac{1}{2}\left(z_{k}^{T} Q_{\mathrm{z}, k} z_{k}+p_{k}^{T} Q_{\mathrm{p}, k} p_{k}\right)
$$

where $Q_{\mathrm{z}, k}$ and $Q_{\mathrm{p}, k}$ are appropriate positive definite symmetric matrices.

We define the functional (cost function) to be minimized over the spatial domain $\mathbb{V}$ with boundary $\mathbb{B}$ subject to dynamic system (9) as follows:

$$
J\left(z_{k}, \mathbf{P}_{k}\right)=\int_{\mathbb{V}} \mathcal{H}\left(z_{k}, \mathbf{P}_{k}\right) \mathrm{d} \xi
$$

where $z_{k}$ is the field initial condition and $\mathbf{P}_{k}$ collects the control actions (control fields) over a control horizon of $M_{\mathrm{c}}$ sampling times, $\mathbf{P}_{k}=\left\{p_{i}\right\}_{i=k}^{k+M_{\mathrm{c}}-1}$. 
Accordingly, $\mathbf{P}_{k}$ produces over the predictive horizon the state sequence $\mathbf{Z}_{k}=$ $\left\{z_{i}\right\}_{i=k+1}^{k+M}$. Function $\mathcal{H}\left(z_{k}, \mathbf{P}_{k}\right)$ in (27) takes the form:

$$
\mathcal{H}\left(z_{k}, \mathbf{P}_{k}\right)=\sum_{i=k}^{k+M-1} h\left(z_{i}, p_{i}\right)+h_{\mathrm{F}}\left(z_{k+M}\right)
$$

with $h_{\mathrm{F}}\left(z_{k+M}\right)=z_{k+M}^{T} Q_{\mathrm{z}, k+M} z_{k+M}$. It is important to remark that since functions $h(z, p)$ and $h_{\mathrm{F}}(z)$ are lower bounded there exists a positive constant $\alpha_{\mathrm{h}}$ such that:

$$
h(z, p) \geqslant \alpha_{\mathrm{h}} z^{T} z, \quad \text { and } \quad h_{\mathrm{F}}(z) \geqslant \alpha_{\mathrm{h}} z^{T} z .
$$

\section{- Optimal Control problem at time $t_{k}$}

The optimal control problem to be solved at every $k$ is that of finding the control profile $\mathbf{P}_{k}^{*}$ over a given control horizon $M_{\mathrm{c}}$ which minimizes the deviation between the state trajectory and the state reference or setpoint over the predictive horizon $M \geqslant M_{\mathrm{c}}$. In this formulation, the plant - Eq. (9) - will be approximated by a number $N$ of slow modes. Each approximation constitutes a model of the plant described by a set of nonlinear differential equations (see Appendix A for details), we formally express as:

$$
\frac{\mathrm{d} m_{\mathrm{A}}}{\mathrm{d} t}=A_{\mathrm{A}} m_{\mathrm{A}}+F_{\mathrm{A}},
$$

with each field is approximated as $z \approx z_{\mathrm{A}}=\sum_{i=1}^{N} \phi_{i} m_{i}$. With some abuse of notation, $m$ should be understood as a vector associated to each field within the vector $z$. Accordingly, a plant approximation would require as many equations of the form (30) as fields considered. The corresponding NMPC 
problem to be solved at every $k$ is then stated as:

$$
\begin{array}{rl}
\min _{\mathbf{P}_{k}} & J\left(z_{k}, \mathbf{P}_{k}\right) \\
\text { s.t. } & \frac{\mathrm{d} m_{\mathrm{A}}}{\mathrm{d} t}=A_{\mathrm{A}} m_{\mathrm{A}}+F_{\mathrm{A}} \\
& z_{k+M}=0 .
\end{array}
$$

Optional constraints may be added to problem (31) for $M_{\mathrm{c}} \leqslant M$. This formulation comprises two types of constraints as described in [50] to enforce the nominal stability of the NMPC controller, the terminal cost term in (28) and the terminal state constraint (31c). At this point we are not considering any additional bounds and/or constraints on the problem solution. Given the NMPC problem with a field initial condition $z_{k}$, we assume that $\mathbf{P}_{k}$ is a feasible solution for (31) and that there exists a sufficiently long horizon that ensures an admissible trajectory to satisfy the terminal constraints. Therefore, from solving (31) we obtain an optimal solution $\mathbf{P}_{k}^{*}$ which when

implemented in the system will produce an optimal state sequence $\mathbf{Z}_{k}^{*}$, and the corresponding optimal objective function value $J\left(z_{k}, \mathbf{P}_{k}^{*}\right)$.

\subsection{Assumptions}

Regarding the developments of the robust stability analysis in the next sections it is essential to list here the main assumptions on which that analysis is based $[49,50]$.

Assumption 2. All the states z can be measured (or estimated) at every time index $k$.

Assumption 3. Both the plant model (9) and the reduced order model (30) 
satisfy $z_{k+1}=0$ for the steady state reference values $\left(z_{k}, p_{k}\right)=(0,0)$. Note that because of cost function definition (26), it follows that $J\left(0, \boldsymbol{P}_{k}^{*}\right)=0$.

Assumption 4. There exists a sufficiently long horizon to ensure an admissible trajectory (i.e., with $z_{k+M}=0$ ). This is a controllability property termed Property $\mathrm{C}$ in [60].

\section{Robustness conditions for Multi-Model Predictive Control}

The stability analysis follows from familiar arguments developed by [61]. We first consider the perfect model case in order to discuss some of the arguments that will be employed to address the mismatch case, resulting from using the reduced order approximation (30).

\subsection{Perfect model case}

Consider the optimal solution of problem (31) at time index $k, \mathbf{P}_{k}^{*}$, with

cost $J\left(z_{k}, \mathbf{P}_{k}^{*}\right)$, and $h_{\mathrm{F}}\left(z_{k+M}\right)=0$, and apply the first element of the optimal control sequence $\left(p_{k}^{*}\right)$. As a result, the system will evolve to a state $z_{k+1}^{*}$ at time index $k+1$. The optimal solution computed in the previous step $\left(\mathbf{P}_{k}^{*}\right)$ is also a feasible candidate for the optimization problem at time index $k+1$ starting from the initial condition $z_{k+1}=z_{k+1}^{*}$. Let $\mathbf{P}_{k+1}^{\prime}=\mathbf{P}_{k}^{*} \backslash p_{k}^{*}$ be the sequence computed at time index $k$ without the first element, and $J\left(z_{k+1}, \mathbf{P}_{k+1}^{\prime}\right)$ be its corresponding cost (which of course might not be the 
optimal one). Then, we can write:

$$
\begin{aligned}
J\left(z_{k}, \mathbf{P}_{k}^{*}\right)-J\left(z_{k+1}, \mathbf{P}_{k+1}^{\prime}\right)= & \int_{\mathbb{V}} \sum_{i=k}^{k+M-1} h\left(z_{i}, p_{i}^{*}\right) \mathrm{d} \xi+\int_{\mathbb{V}} h_{\mathrm{F}}\left(z_{k+M}\right) \mathrm{d} \xi \\
& -\int_{\mathbb{V}} \sum_{i=k+1}^{k+M} h\left(z_{i}, p_{i}^{*}\right) \mathrm{d} \xi-\int_{\mathbb{V}} h_{\mathrm{F}}\left(z_{k+M+1}\right) \mathrm{d} \xi \\
= & \int_{\mathbb{V}} h\left(z_{k}, p_{k}^{*}\right) \mathrm{d} \xi-\int_{\mathbb{V}} h_{\mathrm{F}}\left(z_{k+M+1}\right) \mathrm{d} \xi
\end{aligned}
$$

According to Assumption 4 and Eq (31c) the last term of Eq (32) becomes zero. Thus it simplifies to

$$
J\left(z_{k}, \mathbf{P}_{k}^{*}\right)-J\left(z_{k+1}, \mathbf{P}_{k+1}^{\prime}\right)=\int_{\mathbb{V}} h\left(z_{k}, p_{k}^{*}\right) \mathrm{d} \xi
$$

Consider now the NMPC problem at time index $k+1$. As mentioned above, $\mathbf{P}_{k+1}^{\prime}$ is a feasible candidate for the optimization problem at time index $k+1$, however it might not be the optimal one. Note that, in the worst case, $\mathbf{P}_{k+1}^{*}=\mathbf{P}_{k+1}^{\prime}$ which implies $J\left(z_{k+1}, \mathbf{P}_{k+1}^{*}\right)=J\left(z_{k+1}, \mathbf{P}_{k+1}^{\prime}\right)$. In other words, it is always possible to find a sequence $\mathbf{P}_{k+1}^{*}$ such that $J\left(z_{k+1}, \mathbf{P}_{k+1}^{*}\right) \leqslant$ $J\left(z_{k+1}, \mathbf{P}_{k+1}^{\prime}\right)$. Therefore from (33) it follows that

$$
J\left(z_{k}, \mathbf{P}_{k}^{*}\right)-J\left(z_{k+1}, \mathbf{P}_{k+1}^{*}\right) \geqslant \int_{\mathbb{V}} h\left(z_{k}, p_{k}^{*}\right) \mathrm{d} \xi .
$$

Thus the sequence $J\left(z_{k}, \mathbf{P}_{k}^{*}\right)$ over $n$ time indices decreases, and because $h(\cdot)$ is bounded from below by zero it converges, and $h\left(z_{k}, p_{k}^{*}\right) \rightarrow 0$ with $k \rightarrow n$.

\subsection{Mismatch case}

In order to ensure stability of MPC under mismatch we essentially will follow the arguments given by $[49,50]$. The outcome of the approach will be a criterion on the objective function value of problem (31) which when satisfied it will ensure its decrease along the computed trajectories and therefore 
stability of the closed-loop system. In essence, the criterion will be employed to show that approximations of the slow dynamics for system (9) can always be found such that the system is closed-loop stable in the presence of the plant/reduced order model mismatch.

Assumption 5. In what follows we assume that the optimal control problem at every time index $k$ is feasible in the sense that for any bounded initial condition $z_{k}$ there exists a bounded set of control actions $\boldsymbol{P}_{k}^{*} \equiv \boldsymbol{P}^{*}\left(z_{k}\right)$ which minimizes (31a) subject to (31b) and (31c), being $J\left(z_{k}, \boldsymbol{P}_{k}^{*}\right)$ the minimum of the cost function for the initial condition $z_{k}{ }^{1}$

In this way, for any pair of initial conditions $z_{1, k}$ and $z_{2, k}$ there exists a positive constant $\alpha_{\mathbf{P}}$ such that:

$$
\left\|\mathbf{P}^{*}\left(z_{1, k}\right)-\mathbf{P}^{*}\left(z_{2, k}\right)\right\|_{2}^{2} \leqslant \alpha_{\mathbf{P}}\left\|z_{1, k}-z_{2, k}\right\|_{2}^{2} .
$$

Since function (28) is Lipschitz in its arguments we also have that:

$$
\left|J\left(z_{1, k}, \mathbf{P}^{*}\left(z_{1, k}\right)\right)-J\left(z_{2, k}, \mathbf{P}^{*}\left(z_{2, k}\right)\right)\right| \leqslant \alpha_{\mathbf{J}}\left\|z_{1, k}-z_{2, k}\right\|_{2}^{2} .
$$

With these preliminary observations we are in the position to state next the main robustness condition.

Proposition 2. A predictive controller that makes use of the solution to problem (31) will be stable provided that, for each $k$, the following inequality holds:

$$
J\left(z_{k+1}, \boldsymbol{P}_{k+1}^{*}\right)-J\left(z_{\mathrm{A}, k+1}, \boldsymbol{P}_{\mathrm{A}, k+1}^{*}\right) \leqslant \rho \int_{\mathbb{V}} h\left(z_{k}, p_{k}^{*}\right) \mathrm{d} \xi,
$$

\footnotetext{
${ }^{1}$ Note that the set of optimal control actions must be itself function of the initial condition
} 
where $\rho \in[0,1)$.

Moreover, at each $k$ it is always possible to find a number $N$ of slow modes so to ensure that the above inequality (mismatch condition) holds.

Proof: To prove the first part we add and subtract $J\left(z_{\mathrm{A}, k+1}, \mathbf{P}_{\mathrm{A}, k+1}^{*}\right)$ to the LHS of (34) so that:

$$
\begin{aligned}
J\left(z_{k}, \mathbf{P}_{k}^{*}\right)-J\left(z_{k+1}, \mathbf{P}_{k+1}^{*}\right)= & J\left(z_{k}, \mathbf{P}_{k}^{*}\right)-J\left(z_{\mathrm{A}, k+1}, \mathbf{P}_{\mathrm{A}, k+1}^{*}\right) \\
& -\left(J\left(z_{k+1}, \mathbf{P}_{k+1}^{*}\right)-J\left(z_{\mathrm{A}, k+1}, \mathbf{P}_{\mathrm{A}, k+1}^{*}\right)\right) .
\end{aligned}
$$

Note that since at time index $k$ state measurements are available, $z_{A, k} \equiv z_{k}$. Thus, applying (34) to the first term on the RHS of equation (38) leads to:

$$
\begin{aligned}
J\left(z_{k}, \mathbf{P}_{k}^{*}\right)-J\left(z_{k+1}, \mathbf{P}_{k+1}^{*}\right) \geqslant & \int_{\mathbb{V}} h\left(z_{k}, p_{k}^{*}\right) \mathrm{d} \xi \\
& -\left(J\left(z_{k+1}, \mathbf{P}_{k+1}^{*}\right)-J\left(z_{\mathrm{A}, k+1}, \mathbf{P}_{\mathrm{A}, k+1}^{*}\right)\right) .
\end{aligned}
$$

Combining (37) with (39) we obtain:

$$
J\left(z_{k}, \mathbf{P}_{k}^{*}\right)-J\left(z_{k+1}, \mathbf{P}_{k+1}^{*}\right) \geqslant(1-\rho) \int_{\mathbb{V}} h\left(z_{k}, p_{k}^{*}\right) \mathrm{d} \xi .
$$

Since $h\left(z_{k}, p_{k}^{*}\right)$ is bounded from below by a quadratic function of the field (Eqn (29)) we also have:

$$
J\left(z_{k}, \mathbf{P}_{k}^{*}\right)-J\left(z_{k+1}, \mathbf{P}_{k+1}^{*}\right) \geqslant(1-\rho) \alpha_{h}\left\|z_{k}\right\|_{2}^{2},
$$

what leads to $\lim _{k \rightarrow \infty}\left\|z_{k}\right\|_{2}^{2}=0$, since $1-\rho>0$.

To prove the second part of the proposition we make use of (36) and the orthogonality property of the eigenfunctions to show that the left hand side of (37) is bounded as follows:

$$
\left|J\left(z_{k+1}, \mathbf{P}_{k+1}^{*}\right)-J\left(z_{\mathrm{A}, k+1}, \mathbf{P}_{\mathrm{A}, k+1}^{*}\right)\right| \leqslant \alpha_{\mathbf{J}}\left\|z_{\mathrm{B}, k+1}\right\|_{2}^{2} .
$$


According to proposition $1,\left\|z_{\mathrm{B}, k+1}\right\|_{2}^{2}$ is bounded by its corresponding norm at $k$ as:

$$
\left\|z_{\mathrm{B}, k+1}(T)\right\|_{2}^{2} \leqslant\left\|z_{\mathrm{B}, k}\right\|_{2}^{2} \exp \left(-\varepsilon_{N} T\right)+\frac{2 \beta^{2}}{\varepsilon_{N}^{2}}\left(1-\exp \left(-\varepsilon_{N} T\right)\right)
$$

and the result follows since for any $\left\|z_{\mathrm{B}, k}\right\|_{2}^{2}, \varepsilon_{N} \rightarrow \infty$ as $N \rightarrow \infty$ so the right hand side can be made arbitrarily small by increasing $N$.

\section{The Multi-Model Predictive Control framework}

We demonstrated in the previous section that approximations of the slow dynamics can always be found such that the resulting system is closed-loop stable in the presence of the plant/reduced order model mismatch. Those approximations, however, are not known a priori or even can change depending on the range of operation. In this section, we describe the MMPC methodology to assure close-loop stability and illustrate the main steps with a flow chart (Figure 1).

The basic idea consists of using a given system approximation of the form of equation (31b) to compute the optimal solution of problem (31a). Then, when the sufficient condition for stability described in Proposition 2 (Eq (37)) is not satisfied a new model of the form (30) is recomputed by updating the POD basis. Let us rewrite (37) such that:

$$
J\left(z_{k}, \mathbf{P}_{k}^{*}\right)-J\left(z_{\mathrm{A}, k}, \mathbf{P}_{\mathrm{A}, k}^{*}\right) \leqslant \rho \int_{\mathbb{V}} h\left(z_{k-1}, p_{k-1}^{*}\right) \mathrm{d} \xi .
$$

In order to check this condition, it is necessary to know at any time $t_{k}$ the term in the RHS and the optimal cost function of two optimizations that we denote here as "standard optimization" - since standard MPC also needs its 
calculation - and "non-standard optimization". The only difference between both is the initial condition. In the former such initial condition is the current measure of the field $z_{k}$ while in the "non-standard optimization" it is given by $z_{\mathrm{A}, k}$ which is to be estimated by using the previous field measurement $z_{k-1}$ and the approximation of the system.

In the case that (43) is not satisfied, we need to update the model, i.e., to recalculate the set of PODs representative of the current range of operation. To this purpose we make use of the last $N_{\mathrm{m}}$ measurements obtained from the plant, i.e. $\left\{z_{k-N_{\mathrm{m}}}, \ldots, z_{k}\right\}$. The technique to re-calculate the PODs is outlined in Appendix A, and the criterion to choose the dimension of the POD set is determined by the percentage of energy captured by the approximation. It must be highlighted that considering different sets of snapshots will, in general, lead to different sets of PODs. Therefore, the number of PODs necessary to capture a given percentage of energy will depend on the considered set of snapshots. A nominal criterion for the energy captured could be $99 \%$ as proposed by [38]. A good selection of number of measurements, $N_{\mathrm{m}}$, and the energy captured by the PODs, $E(\%)$, could accelerate considerably the computation of the optimal profiles. When $N_{\mathrm{m}}$ and $E(\%)$ are small, numerous updates must be done. On the contrary, when these indices are large, few updates of the model are required but its dimension is higher. Consequently it leads to an increase of the computational effort to solve the optimization problem.

Simulation studies with the system described in Section 6, indicate that these parameters must be selected with a compromise between the quality of the model approximation and the computational cost to solve the optimiza- 
tion problem.

When implementing this technique, there are also other important aspects which will speed up substantially the calculations (see Figure 1). For example, the RHS term required at time $t_{k}$ in (43) is implicitly calculated by the "standard optimization" at time $t_{k-1}$. The schematic representation of the algorithm in Figure 1 gives also the basic steps to follow taking into account scenarios where numerical errors may result in unnecessary updates of the model. Note that when the plant is close to the reference point, the terms in (43) are close to zero and therefore very small values may trigger unnecessary model updates. As it is illustrated in Section 6, this can be avoided by permitting model updates only when the cost function value is higher than a given tolerance $\epsilon$, i.e.,

$$
J\left(z_{k}, \mathbf{P}_{\mathrm{A}, k}^{*}\right)>\epsilon
$$

Finally, we recall that in this work we focus on nonlinear diffusion-convectionreaction systems, and that their dissipative nature ensures the existence of a reduced order representation capable of approximating at arbitrary accuracy the real plant dynamics for a given operation region. Nevertheless, we emphasize that it is not possible to define precisely the typical range of $N$ values for this class of systems. Previous works on this class of systems $([62,32,54,53])$ show that for most systems the range of the number of ODEs per field is not larger than $10^{2}$ (even for the most complex hydrodynamic systems in $2 \mathrm{D}$ or 3D) in order to obtain a reasonably accurate description of the dynamics. A priori, this range of values renders NMPC practical. Among other aspects to take into consideration regarding the practicability of NMPC are the formulation, algorithm, and numerical implementation strategies. For example, 
[27] report the application of their advanced-step NMPC to a distributed system described by a DAE model with 350 states and differential-algebraic equations. Thus, the adoption of the reduced order methodology described in this work can contribute to the application of existing advanced NMPC technology to distributed systems of higher dimension.

\section{Case study}

The performance of the multimodel MPC approach is demonstrated by simulation experiments on a non-isothermal tubular reactor with recycle. The reactant concentration, $C \equiv C(\xi, t)$, and the temperature inside the reactor, $T \equiv T(\xi, t)$, are described by the following set of PDEs $[63,64]$ :

$$
\begin{aligned}
& \frac{\partial C}{\partial t}=\frac{1}{\mathrm{Pe}_{C}} \frac{\partial^{2} C}{\partial \xi^{2}}-\frac{\partial C}{\partial \xi}-f(C, T), \\
& \frac{\partial T}{\partial t}=\frac{1}{\mathrm{Pe}_{T}} \frac{\partial^{2} T}{\partial \xi^{2}}-\frac{\partial T}{\partial \xi}+\mathrm{B}_{T} f(C, T)+\beta_{T}\left(T_{\mathrm{c}}-T\right),
\end{aligned}
$$

where $T_{\mathrm{c}}$ is the control variable and represents the cooling jacket temperature which is assumed to be uniform along the reactor. The nonlinear kinetic term $f(C, T)$ is given by

$$
f(C, T)=\mathrm{B}_{C}(1+C) \exp \left(\frac{\gamma T}{1+T}\right) .
$$

The reactant of the outlet stream is recycled to the reactor feed at a ratio $r$ which results into the following boundary conditions:

$$
\begin{aligned}
& \xi=0:\left\{\begin{array}{l}
\frac{\partial C}{\partial \xi}=\operatorname{Pe}_{C}\left[(1-r) C_{0}+r C(L, t)-C(0, t)\right] \\
\frac{\partial T}{\partial \xi}=\operatorname{Pe}_{T}\left[(1-r) T_{0}+r T(L, t)-T(0, t)\right]
\end{array},\right. \\
& \xi \equiv L=1: \frac{\partial C}{\partial \xi}=\frac{\partial T}{\partial \xi}=0 .
\end{aligned}
$$


The values of the parameters are chosen from $[63,65]$ :

$$
\begin{array}{lll}
\mathrm{Pe}_{C}=7, & \mathrm{~B}_{C}=0.1, & \gamma=10, \\
\mathrm{Pe}_{T}=7, & \mathrm{~B}_{T}=2.5, & \beta_{T}=2 .
\end{array}
$$

Under the operating conditions $C_{0}=T_{0}=0, T_{\mathrm{c}}=0$, and with a recycle ratio $r=0.5$, the reactor exhibits limit cycle behavior (Figure 2). The objective of the MMPC strategy (Figure 1) is to stabilize the limit cycle around a given outlet concentration setpoint, $C_{\mathrm{out}}^{\mathrm{sp}}$, using $T_{\mathrm{c}}$ as the manipulated input. The objective function consists of a weighted quadratic cost function with two terms. One term is defined as the square of the deviation between the output concentration, $C_{\text {out }} \equiv C(L, t)$, and the concentration setpoint, and the other one penalizes the square of deviation of $T_{\mathrm{c}}$ with respect to a given reference value $T_{\mathrm{c}}^{\mathrm{ref}}$. The optimal control problem becomes:

$$
\begin{aligned}
& \min _{T_{\mathrm{c}}} . J_{k}= \sum_{i=k}^{k+M-1}\left[\alpha\left(C_{\mathrm{out}, i}-C_{\mathrm{out}, i}^{\mathrm{sp}}\right)^{2}+\beta\left(T_{\mathrm{c}, i}-T_{\mathrm{c}, i}^{\mathrm{ref}}\right)^{2}\right] \\
& \text { s.t. } \frac{\mathrm{d} m_{\mathrm{A}}^{C}}{\mathrm{~d} t}=\int_{\mathbb{V}} \phi_{\mathrm{A}}^{C}\left(\frac{1}{\mathrm{Pe}_{C}} \frac{\partial^{2} C}{\partial \xi^{2}}-\frac{\partial C}{\partial \xi}-f(C, T)\right) \mathrm{d} \xi \\
& \frac{\mathrm{d} m_{\mathrm{A}}^{T}}{\mathrm{~d} t}=\int_{\mathbb{V}} \phi_{\mathrm{A}}^{T}\left(\frac{1}{\mathrm{Pe}_{T}} \frac{\partial^{2} T}{\partial \xi^{2}}-\frac{\partial T}{\partial \xi}+\mathrm{B}_{T} f(C, T)+\beta_{T}\left(T_{\mathrm{c}}-T\right)\right) \mathrm{d} \xi
\end{aligned}
$$

where $\alpha$ and $\beta$ are the weighting parameters, and $m_{\mathrm{A}}^{x}=\left[m_{1}^{x}, m_{2}^{x}, \ldots, m_{N_{x}}^{x}\right]^{T}$, $\phi_{\mathrm{A}}^{x}=\left[\phi_{1}^{x}, \phi_{2}^{x}, \ldots, \phi_{N_{x}}^{x}\right]^{T}, x=[C, T]$. We consider in this example that $C_{\mathrm{out}, k}^{\mathrm{sp}}=$ -0.9 and $T_{\mathrm{c}, k}^{\mathrm{ref}}=-0.01$ for every $t_{k}$ although other values (even a time dependent function) could be also considered for $C_{\mathrm{out}, k}^{\mathrm{sp}}$. The weighting parameters in (46a) are $\alpha=100$ and $\beta=100$.

In this simulation experiment the real plant will consist of a finite element implementation of model (45) using a uniform spatial grid. The degree 
of discretization (31 nodes in this example) is selected such as further discretizations do not significantly alter the solution. The model employed to approximate the plant behavior is constructed using the POD technique (see (46b)-(46c)) where the number of slow modes in the approximation $\left(N_{C}, N_{T}\right)$ is computed using the energy criteria (see Appendix A for more details). Some relevant aspects of the MMPC implementation are outlined next (see also Figure 1):

1. At the beginning of the process operation there are no snapshots available to derive the POD basis. Hence, the reactor operates in open-loop mode until $t=1$, and several snapshots are taken during this period of time. It should be noted that this is a limitation of the methodology that can be mitigated by building an off-line reduced model based on measurements of a similar plant or on data obtained from a model available in the literature.

2. Using these snapshots the POD basis that captures $99.9 \%$ of the energy is computed and the ROM is This value of energy was selected to compensate for the poor information available from the snapshots at this point.

3. This ROM is employed to solve the nonlinear MPC problem (46a) with a predictive horizon $M=6$ and control horizon $M_{\mathrm{c}}=2$. It is noted that long predictive horizons are required to satisfy Assumption 4. However, as it will be shown, short horizons can be used as well. In our case study, off-line tests of the optimal control problem were carried out to estimate such values.

4. New concentration and temperature measurements are taken at every 
sampling time, $\Delta t=0.5$ time units.

5. Before each new optimization problem, criteria (43) and (44) are checked. In case they do not fulfill, new POD basis that captures $99.5 \%$ of the energy is computed using the snapshots obtained from the measurements in step 4, and a new ROM is derived. Note that the value of energy in this step is lower than that one considered in step 2 because a richer information (snapshots) is available at this point. Also it was selected larger than the one recommended in [38] because it ensures a good representation of the plant behavior while keeping the model dimensionality adequate for MPC purposes.

6. Steps 3-5 are repeated.

It must be noted that from a practical implementation point of view, the solution of the optimal control problem should remain small as compared to the process time constants for the controller to operate properly. This implies a ratio $\ell / v$ large enough as compared with the time to complete each optimization $^{2}$. In our case, where optimizations where performed in the order of 10 seconds, ratios of about 100 seconds should suffice.

First of all, in order to emphasize the importance of the updating procedure, a conventional MPC scheme (which employs a ROM of the form (30) without model updating) was applied. It must be noted that since the model employed in this scheme may not be accurate enough to represent the oscillatory behavior of the plant (due to, for instance, not enough process information is available), the conventional MPC scheme is neither able to

\footnotetext{
${ }^{2} \ell$ and $v$ correspond with physical reactor length and fluid velocity, respectively
} 
target the concentration setpoint nor to stabilize the system. This is the case illustrated in Figures 3 and 5. Alternatively a more representative sampling could capture the precise behavior but at the expenses of exhaustive off-line experiments to be carried out beforehand.

On the contrary, the proposed MMPC methodology is able to detect through (43) and (44) when the current ROM is not accurate enough to track the outlet concentration setpoint producing new representative POD sets in the sense of ensuring controller stability. The circle marks in Figures 5 and 6 indicate when the model was updated using the new available state measurements. Figures 4 and 5 show that the MMPC approach is able to stabilize the system around the desired outlet concentration setpoint. Four model updates were required, respectively at $t=3,10,12$, and 12.5 units of time. It is important to note that the ROM obtained with $99.5 \%$ of the energy always consisted of less than 6 ODEs per field.

Finally, the evolution of objective function (46a) as well as inequality (43) are represented in Figure 6 illustrating that, for the MMPC approach, asymptotic convergence is ensured. Besides, the evolution of the mismatch term, expressed in terms of the $L_{2}$ norm of the differences between the measured (real) states, $y=\left[C_{\text {out }}, T_{\text {out }}\right]$, and the predicted states, $\hat{y}=\left[\hat{C}_{\text {out }}, \hat{T}_{\text {out }}\right]$ ) is also represented in Figure 6. When applying the conventional MPC scheme this term keeps oscillating whereas the MMPC approach is able to reduce it to zero. 


\section{Conclusions}

Robustness in MPC is at a high extent conditioned by the available model of the plant on which the accuracy of predictions rely. It is well known that although process-model mismatch can lead to serious control performance deterioration, a certain amount can be tolerated in the sense that it will not destroy the stability of the control loop. However the question is on how to determine such a bound on the allowable amount of mismatch. In this work we have shown that a positive answer to this question is possible at least for dissipative systems as those described by convection-diffusion-reaction mechanisms.

In that purpose, arguments similar as those proposed by $[49,50]$ have been employed to demonstrate that it is always possible to choose within a collection of reduced approximations (models) one that preserves the stabilizing properties of MPC despite uncertainties and disturbances.

The practical implication of this evidence is a robust nonlinear model predictive controller that makes use of a collection of reduced order approximations of the plant (models) reconstructed on-line by projection methods on POD (Proper Orthogonal Decomposition) basis functions. Since plant approximations are built on-line based on actual measurements the proposed controller can be interpreted as a multi-model nonlinear MPC or MMPC.

The performance of the MMPC strategy has been illustrated by simulation experiments on a problem that involves temperature and concentration control of a tubular reactor with recycle. Extensions of this methodology to more general classes of systems can be foreseen as long as their representations can be cast into complete sets. 


\section{Acknowledgments}

This work has been also partially founded by the Spanish Ministry of Science and Innovation (SMART-QC, AGL2008-05267-C03-01), the FP7 CAFE project (KBBE-2007-1-212754), the Project PTDC/EQU-ESI/73458/2006 from the Portuguese Foundation for Science and Technology and PI grant 07/IN.1/I1838 by Science Foundation Ireland. Also, the authors acknowledge financial support received by a collaborative grant GRICES-CSIC. Finally, we want to thank the reviewers for their useful comments.

\section{Appendix A. Reduced order models}

Let us start the derivation of reduce order models (ROM) by expanding the field into a Fourier series of the form (6):

$$
z(t, \xi)=\sum_{i=1}^{\infty} m_{i}(t) \phi_{i}(\xi)
$$

where $\left\{\phi_{i}(\xi)\right\}_{i=1}^{\infty}$ is the set of basis functions containing the spatial information of the solution. The time information is encoded in the so-called mode set $\left\{m_{i}(t)\right\}_{i=1}^{\infty}$. Each element $\phi_{i}(\xi)$ of the set of basis functions is computed off-line as the solution of the following integral eigenvalue problem $[38,63,66]$ :

$$
\lambda_{i} \int_{\mathbb{V}} R\left(\xi, \xi^{\prime}\right) \phi_{i}\left(\xi^{\prime}\right) \mathrm{d} \xi^{\prime}=\phi_{i}(\xi)
$$

where $\lambda_{i}$ corresponds with the eigenvalue associated with each global eigenfunction $\phi_{i}$. In this work, the proper orthogonal decomposition (POD) will be selected as the technique to compute the basis functions due to its efficiency [54]. In the POD, the kernel $R\left(\xi, \xi^{\prime}\right)$ in equation (A.2) corresponds 
with the two point spatial correlation function, defined as follows [54]:

$$
R\left(\xi, \xi^{\prime}\right)=\frac{1}{\ell} \sum_{j=1}^{\ell} z\left(\xi, t_{j}\right) z\left(\xi^{\prime}, t_{j}\right) .
$$

where $z\left(\xi, t_{j}\right)$ corresponds with the value of the field at each instant $t_{j}$ and the summation extends over a sufficiently rich collection of uncorrelated snapshots at $j=1, \cdots, \ell[38]$. The basis functions obtained by means of the POD technique are also known as empirical basis functions or POD basis.

The dissipative nature of the kind of systems considered in this work allows us to separate the system dynamics in two subsets [67, 68, 31]: one stable with fast dynamics and infinite dimensional $\left(z_{B}\right)$ and the other $\left(z_{\mathrm{A}}\right)$ composed by a finite number of elements with slow dynamics which may be unstable and will capture the relevant dynamics of the system. The number of elements $(N)$ in the stable subset is usually chosen using a criteria based on the energy captured by the POD basis. Such energy is connected to the eigenspectrum $\left\{\lambda_{i}\right\}_{i=1}^{\ell}$ or, to be more precise, to the inverse of the eigenvalues $\mu_{i}=1 / \lambda_{i}$ as follows (for a deeper insight see $[63,38]$ ):

$$
E(\%)=100 \times \frac{\sum_{i=1}^{N} \mu_{i}}{\sum_{i=1}^{\ell} \mu_{i}}
$$

Note that $\mu_{i} \rightarrow 0$ as $i \rightarrow \infty$ since, as pointed out in section $2.1, \lambda_{i} \rightarrow \infty$ as $i \rightarrow \infty$.

In this work, the numerical solution of Eqn (A.2) has been computed by taking advantage of the finite element structure. A detailed explanation of such procedure is provided in [69]. It is worth mentioning that the computational time for solving the eigenvalue problem (A.2) rapidly increase with 
the number of discretization points. For most of $1 \mathrm{D}$ problems this should not be an issue. However, when considering 2D or 3D systems, solving (A.2) may render impractical for MPC purposes. In those cases, the method of snapshots or strobes proposed in [38] can be employed.

Finally, in order to recover the field $z_{\mathrm{A}}(\xi, t)$, the original PDE system (9) is projected onto the slow basis set $^{3}$. As a result, the following set of ODEs is obtained:

$$
\frac{\mathrm{d} m_{\mathrm{A}}}{\mathrm{d} t}=A_{\mathrm{A}} m_{\mathrm{A}}+F_{\mathrm{A}}
$$

where $m_{\mathrm{A}}=\left[m_{1}, m_{2}, \cdots, m_{N}\right]^{T}, A_{\mathrm{A}}=\left\langle\phi_{\mathrm{A}}, \frac{\partial}{\partial x}\left(k \frac{\partial \phi_{\mathrm{A}}}{\partial x}\right)-\mathrm{v} \frac{\partial \phi_{\mathrm{A}}}{\partial x}\right\rangle_{\mathbb{V}}, F_{\mathrm{A}}=$ $\left\langle\phi_{\mathrm{A}}, f(z)+p(x, t)\right\rangle_{\mathbb{V}}$ and $\phi_{\mathrm{A}}=\left[\phi_{1}, \phi_{2}, \cdots, \phi_{N}\right]^{T}$.

It is important to highlight that, as shown in section 2 , the number of elements $N$ in the slow subset $z_{\mathrm{A}}$ can be increased to approximate the original state $z$ with an arbitrary degree of accuracy.

\section{Appendix B. Boundedness of the convective term}

In order to show the conditions under which the first derivative can be expanded in convergent series of the form (13) let us start with a definition:

Definition 1 (Passive systems). A system is said to be passive if there exists a function $\mathcal{W}(t)$ bounded from below so that

$$
\mathcal{W}(T+t)-\mathcal{W}(t) \geqslant \int_{t}^{T+t}\langle y, p\rangle_{\mathbb{V}} \quad \forall t, T>0
$$

with $p$ and $y$ being, respectively, the input and the output of the system.

\footnotetext{
${ }^{3}$ The basis set has been previously normalized so that $\int_{\mathbb{V}} \phi_{i} \phi_{i} \mathrm{~d} \xi=1, \forall i=1, . ., N$.
} 
Consider the quadratic function $\mathcal{W}(t)=\frac{1}{2}\langle z, z\rangle_{\mathbb{V}}=\frac{1}{2}\|z\|_{2}^{2}$. Multiplying Eq (9) by $z$ and integrating over the spatial coordinates, we obtain:

$$
\frac{\mathrm{d} \mathcal{W}}{\mathrm{d} t}=\left\langle z, \frac{\partial}{\partial \xi}\left(k \frac{\partial z}{\partial \xi}\right)\right\rangle_{\mathbb{V}}-\left\langle z, v \frac{\partial z}{\partial \xi}\right\rangle_{\mathbb{V}}+\langle z, f\rangle_{\mathbb{V}}+\langle z, p\rangle_{\mathbb{V}}
$$

The first two terms on the RHS of Eq (B.2) were proved to be non positive in [55], therefore using $\mathrm{Eq}(5)$ with $\mu=0$ leads to:

$$
\frac{\mathrm{d} \mathcal{W}}{\mathrm{d} t} \leqslant-L_{0}+\langle z, p\rangle_{\mathbb{V}}
$$

Taking into account that $L_{0}$ is a positive quantity, time integration of Eq (B.3) yields:

$$
\mathcal{W}(T) \leqslant \mathcal{W}(0)+\int_{0}^{T}\langle z, p\rangle_{\mathbb{V}} \mathrm{d} s
$$

Note that this inequality implies that $\mathcal{W}(T)$ is upper bounded for all $T>0$ provided that the controls are also bounded so that $\langle z, p\rangle_{\mathbb{V}}<\infty$.

Let us now consider the diffusive term in Eq (B.2):

$$
\int_{\mathbb{V}} \frac{\partial}{\partial x}\left(z k \frac{\partial z}{\partial \xi}\right) \mathrm{d} \xi=\int_{\mathbb{V}} \frac{\partial z}{\partial \xi} k \frac{\partial z}{\partial \xi} \mathrm{d} \xi+\left\langle z, \frac{\partial}{\partial \xi}\left(k \frac{\partial z}{\partial \xi}\right)\right\rangle_{\mathbb{V}}
$$

or, by means of the divergence theorem

$$
\int_{\mathbb{B}} n\left(z k \frac{\partial z}{\partial \xi}\right) \mathrm{d} \xi=\int_{\mathbb{V}} \frac{\partial z}{\partial \xi} k \frac{\partial z}{\partial \xi} \mathrm{d} \xi+\left\langle z, \frac{\partial}{\partial \xi}\left(k \frac{\partial z}{\partial \xi}\right)\right\rangle_{\mathbb{V}}
$$

It should be noted that, making use of boundary condition (3), the LHS is negative and we obtain

$$
\begin{gathered}
-\int_{\mathbb{V}} \frac{\partial z}{\partial \xi} k \frac{\partial z}{\partial \xi} \mathrm{d} \xi \geqslant\left\langle z, \frac{\partial}{\partial \xi}\left(k \frac{\partial z}{\partial \xi}\right)\right\rangle_{\mathbb{V}} \Rightarrow \\
-k\left\|\frac{\partial z}{\partial \xi}\right\|_{2}^{2} \geqslant\left\langle z, \frac{\partial}{\partial \xi}\left(k \frac{\partial z}{\partial \xi}\right)\right\rangle_{\mathbb{V}}
\end{gathered}
$$


Substituting expression (B.5) into (B.2) and using the Lipschitz condition (5) with $\mu=0$ :

$$
\frac{\mathrm{d} \mathcal{W}}{\mathrm{d} t} \leqslant-k\left\|\frac{\partial z}{\partial \xi}\right\|_{2}^{2}-\left\langle z, v \frac{\partial z}{\partial \xi}\right\rangle_{\mathbb{V}}-L_{0}+\langle z, p\rangle_{\mathbb{V}}
$$

Taking into account that $\left\langle z, v \frac{\partial z}{\partial \xi}\right\rangle_{\mathbb{V}} \geqslant 0-$ see Eqn (17)-

$$
\frac{\mathrm{d} \mathcal{W}}{\mathrm{d} t} \leqslant-k\left\|\frac{\partial z}{\partial \xi}\right\|_{2}^{2}-L_{0}+\langle z, p\rangle_{\mathbb{V}}
$$

Finally, time integration of the former expression leads to:

$$
\int_{0}^{T} k\left\|\frac{\partial z}{\partial \xi}\right\|_{2}^{2} \mathrm{~d} s \leqslant \mathcal{W}(0)-\mathcal{W}(T)+\int_{0}^{T}\left(-L_{0}+\langle z, p\rangle_{\mathbb{V}}\right) \mathrm{d} s
$$

Since the all the terms in the RHS are upper bounded - see Eq. (B.4) - we conclude that the first derivative is bounded.

\section{References}

[1] J. Richalet, A. Rault, J. L. Testud, J. Papon, Model predictive heuristic control: Applications to industrial processes, Automatica 14 (5) (1978) 413-428.

[2] C. R. Cutler, B. L. Ramaker, Dynamic matrix control - a computer algorithm, in: 86th National Meeting of the American Institute of Chemical Engineers, Houston, TX, 1979, 1979, pp. 13-15.

[3] S. Qin, T. Badgwell, A survey of industrial model predictive control technology, Control Engineering Practice 11 (7) (2003) 733-764. 
[4] E. F. Camacho, C. Bordons, Model Predictive Control, 2nd Edition, Advanced Textbooks in Control and Signal Processing, Springer-Verlag, London, 2004.

[5] J. B. Rawlings, D. Q. Mayne, Model Predictive Control: Theory and Design, Nob Hill Publishing, LLC, 2009.

[6] W. H. Ray, Multivariable process control - a survey, Computers \& Chemical Engineering 7 (4) (1983) 367 - 394.

[7] C. García, D. Prett, M. Morari, Model predictive control: Theory and practice a survey, Automatica 25 (3) (1989) 335-348.

[8] M. Morari, J. H. Lee, Model predictive control: Past, present and future, Computers \& Chemical Engineering 23 (4-5) (1999) 667-682.

[9] A. Bemporad, M. Morari, Robust model predictive control: A survey, in: A. Tesi, A. Vicino (Eds.), Robustness in Identification and Control, no. 245 in Lecture Notes in Control and Information Sciences, SpringerVerlag, 1999, pp. 207-226.

[10] J. Rawlings, Tutorial overview of model predictive control, Control Systems Magazine, IEEE 20 (3) (2000) 38-52.

[11] B. Bequette, Nonlinear control of chemical processes: A review, Industrial \& Engineering Chemistry Research 30 (7) (1991) 1391-1413.

[12] M. Henson, Nonlinear model predictive control: current status and future directions, Computers and Chemical Engineering 23 (2) (1998) 187202. 
[13] M. Cannon, Efficient nonlinear model predictive control algorithms, Annual Reviews in Control 28 (2) (2004) 229-237.

[14] L. Magni, D. M. Raimondo, F. Allgöwer (Eds.), Nonlinear Model Predictive Control - Towards New Challenging Applications, Vol. 384 of Lecture Notes in Control and Information Sciences, Springer, 2009.

[15] S. Qin, T. Badgwell, An overview of nonlinear model predictive control applications, Nonlinear model predictive control (2000) 369-392.

[16] D. Q. Mayne, J. B. Rawlings, C. V. Rao, P. O. M. Scokaert, Constrained model predictive control: Optimality and stability, Automatica 36 (6) (2000) 789-814.

[17] D. Limon, T. Alamo, F. Salas, E. Camacho, Input to state stability of min-max mpc controllers for nonlinear systems with bounded uncertainties, Automatica 42 (5) (2006) 797-803.

[18] D. Limon, I. Alvarado, T. Alamo, E. Camacho, Robust tube-based mpc for tracking of constrained linear systems with additive disturbances, Journal of Process Control 20 (3) (2010) 248-260.

[19] A. A. Patwardhan, G. T. Wright, T. F. Edgar, Nonlinear modelpredictive control of distributed-parameter systems, Chemical Engineering Science 47 (4) (1992) 721 - 735.

[20] G. T. Wright, T. F. Edgar, Nonlinear model predictive control of a fixed-bed water-gas shift reactor: An experimental study, Computers \& Chemical Engineering 18 (2) (1994) 83-102. 
[21] N. Padhiyar, S. Bhartiya, Profile control in distributed parameter systems using lexicographic optimization based MPC, Journal of Process Control 19 (1) (2009) $100-109$.

[22] P. Dufour, Y. Touré, D. Blanc, P. Laurent, On nonlinear distributed parameter model predictive control strategy: on-line calculation time reduction and application to an experimental drying process, Computers \& Chemical Engineering 27 (11) (2003) 1533 - 1542.

[23] P. Dufour, Y. Touré, Multivariable model predictive control of a catalytic reverse flow reactor, Computers \& Chemical Engineering 28 (11) (2004) $2259-2270$.

[24] J. De Temmerman, P. Dufour, B. Nicolaï, H. Ramon, MPC as control strategy for pasta drying processes, Computers \& Chemical Engineering $33(1)(2009) 50-57$.

[25] E. Aggelogiannaki, H. Sarimveis, D. Koubogiannis, Model predictive control in long ducts by means of a neural network approximation tool, Applied Thermal Engineering 27 (14-15) (2007) 2363 - 2369.

[26] E. Aggelogiannaki, H. Sarimveis, Nonlinear model predictive control for distributed parameter systems using data driven artificial neural network models, Computers \& Chemical Engineering 32 (6) (2008) 12251237.

[27] V. M. Zavala, L. T. Biegler, Optimization-based strategies for the operation of low-density polyethylene tubular reactors: nonlinear model 
predictive control, Computers \& Chemical Engineering 33 (10) (2009) $1735-1746$.

[28] C. Antoniades, P. D. Christofides, Integrated optimal actuator/sensor placement and robust control of uncertain transport-reaction processes, Computers \& Chemical Engineering 26 (2) (2002) 187-203.

[29] J. A. Burns, B. B. King, A reduced basis approach to the design of loworder feedback controllers for nonlinear continuous systems, Journal of Vibration and Control 4 (3) (1998) 297 - 323.

[30] C. W. Gear, I. G. Kevrekidis, C. Theodoropoulos, "coarse" integration/bifurcation analysis via microscopic simulators: micro-galerkin methods, Computers \& Chemical Engineering 26 (7-8) (2002) 941-963.

[31] A. A. Alonso, I. G. Kevrekidis, J. R. Banga, C. E. Frouzakis, Optimal sensor location and reduced order observer design for distributed process systems, Computers and Chemical Engineering 28 (1-2) (2004) 27-35.

[32] A. A. Alonso, C. V. Fernández, J. R. Banga, Dissipative systems: from physics to robust nonlinear control, Int. J. Robust Nonlinear Control 14 (2) (2004) 157-179.

[33] D. Zheng, K. A. Hoo, Low-order model identification for implementable control solutions of distributed parameter systems, Computers \& Chemical Engineering 26 (7-8) (2002) 1049-1076.

[34] D. Zheng, K. A. Hoo, System identification and model-based control for distributed parameter systems, Computers \& Chemical Engineering 28 (8) (2004) 1361-1375. 
[35] H. Shang, J. F. Forbes, M. Guay, Model predictive control for quasilinear hyperbolic distributed parameter systems, Industrial and Engineering Chemistry Research 43 (9) (2004) 2140-2149.

[36] S. Dubljevic, P. D. Christofides, Predictive control of parabolic PDEs with boundary control actuation, Chemical Engineering Science 61 (18) (2006) $6239-6248$.

[37] S. Dubljevic, Model predictive control of kuramoto-sivashinsky equation with state and input constraints, Chemical Engineering Science 65 (15) (2010) $4388-4396$.

[38] L. Sirovich, Turbulence and the dynamics of coherent structures. Part I: Coherent structures, Quaterly of Appl. Math. 45 (3) (1987) 561-571.

[39] G. Berkooz, P. Holmes, L. Lumley, The Proper Orthogonal Decomposition in the analysis of turbulent flows, Ann. Rev. Fluid Mech. 25 (1) (1993) 539-575.

[40] P. Holmes, J. L. Lumley, G. Berkooz, Turbulence, Coherent Structures, Dynamical Systems and Symmetry., Cambridge University Press, 1996.

[41] P. J. Holmes, J. L. Lumley, G. Berkooz, J. C. Mattingly, R. W. Wittenberg, Low-dimensional models of coherent structures in turbulence, Physics Reports 287 (4) (1997) 337-384.

[42] R. Padhi, S. F. Ali, An account of chronological developments in control of distributed parameter systems, Annual Reviews in Control 33 (1) (2009) 59-68. 
[43] W. Xie, C. Theodoropoulos, An off-line model reduction-based technique for on-line linear mpc applications for nonlinear large-scale distributed systems, Computer Aided Chemical Engineering 28 (2010) 409414.

[44] S. Shvartsman, C. Theodoropoulos, R. Rico-Martinez, I. Kevrekidis, E. Titi, T. Mountziaris, Order reduction for nonlinear dynamic models of distributed reacting systems, Journal of Process Control 10 (2-3) (2000) $177-184$.

[45] C. Theodoropoulos, Optimisation and linear control of large scale nonlinear systems: a review and a suite of model reduction-based techniques, Coping with Complexity: Model Reduction and Data Analysis (2011) 37-61.

[46] K. Veroy, A. Patera, Certified real-time solution of the parametrized steady incompressible navier-stokes equations: rigorous reduced-basis a posteriori error bounds, International Journal for Numerical Methods in Fluids 47 (8-9) (2005) 773-788.

[47] J. Atwell, B. King, Proper orthogonal decomposition for reduced basis feedback controllers for parabolic equations, Mathematical and computer modelling 33 (1-3) (2001) 1-19.

[48] J. Atwell, B. King, Reduced order controllers for spatially distributed systems via proper orthogonal decomposition, SIAM Journal on Scientific Computing 26 (2004) 128. 
[49] L. O. Santos, L. T. Biegler, A tool to analyze robust stability for model predictive controllers, Journal of Process Control 9 (11) (1999) 233-246.

[50] L. Santos, L. Biegler, J. Castro, A tool to analyze robust stability for constrained nonlinear mpc, Journal of Process Control 18 (3-4) (2008) 383-390.

[51] M. R. García, Identification and real time optimization in the food processing and biotechnology industries, Ph.D. thesis, University of Vigo "http://digital.csic.es/bitstream/10261/4662/1/ MiriamRGarcia_thesis.pdf" (2008).

[52] M. R. García, C. Vilas, L. O. Santos, A. A. Alonso, A robust and stabilizing multi-model predictive control approach to command the operation of distributed process systems, in: AIChE 2006 Anual Meeting, San Francisco, CA, 2006, p. 455b.

[53] C. Vilas, A. V. Wouwer, Combination of multi-model predictive control and the wave theory for the control of simulated moving bed plants, Chemical Engineering Science 66 (4) (2011) 632-641.

[54] M. R. García, C. Vilas, J. R. Banga, A. A. Alonso, Optimal field reconstruction of distributed process systems from partial measurements, Industrial \& Engineering Chemistry Research 46 (2) (2007) 530-539.

[55] C. Vilas, M. R. García, J. R. Banga, A. A. Alonso, Robust feed-back control of distributed chemical reaction systems, Chemical Engineering Science 62 (11) (2007) 2941-2957. 
[56] A. A. Alonso, B. E. Ydstie, Stabilization of distributed systems using irreversible thermodynamics, Automatica 37 (11) (2001) 1739-1755.

[57] B. Reddy, Introductory functional analysis: with applications to boundary value problems and finite elements, Springer Verlag, 1998.

[58] J. Smoller, Shock Waves and Reaction-Diffusion Equations, 2nd Edition, Springer-Verlag, New york, 1994.

[59] E. Sontag, The ISS philosophy as a unifying framework for stability-like behavior, Vol. 259 of Lecture Notes in Control and Information Sciences, CNRS, 2001, pp. 443-467.

[60] S. S. Keerthi, E. G. Gilbert, Optimal infinite-horizon feedback laws for general class of constrained discrete-time systems: Stability and movinghorizon approximations, Journal of Optimization Theory and Applications 57 (2) (1988) 265-293.

[61] J. B. Rawlings, K. R. Muske, The stability of constrained receding horizon control, Automatic Control, IEEE Transactions on 38 (10) (1993) 1512-1516.

[62] E. Balsa-Canto, A. A. Alonso, J. R. Banga, Reduced-order models for nonlinear distributed process systems and their application in dynamic optimization, Industrial \& Engineering Chemistry Research 43 (13) (2004) 3353-3363.

[63] A. A. Alonso, C. E. Frouzakis, I. G. Kevrekidis, Optimal sensor placement for state reconstruction of distributed process systems, AIChE Journal 50 (7) (2004) 1438-1452. 
[64] C. Antoniades, P. D. Christofides, Integrating nonlinear output feedback control and optimal actuator/sensor placement for transport-reaction processes, Chemical Engineering Science 56 (15) (2001) 4517-4535.

[65] C. Antoniades, P. D. Christofides, Studies on nonlinear dynamics and control of a tubular reactor with recycle, Nonlinear Analysis 47 (9) (2001) 5933-5944.

[66] S. S. Ravindran, A reduced-order approach for optimal control of fluids using proper orthogonal decomposition, International journal for numerical methods in fluids 34 (5) (2000) 425-448.

[67] C. Antoniades, P. D. Christofides, Computation of optimal actuator locations for nonlinear controllers in transport reaction processes, Comp. Chem. Eng. 24 (2-7) (2000) 577-583.

[68] P. D. Christofides, Nonlinear and Robust Control of PDE Systems: Methods and Applications to Transport-Reaction Processes, Birkhäuser, Boston, 2001.

[69] M. García, C. Vilas, J. Banga, A. Alonso, Exponential observers for distributed tubular (bio) reactors, AIChE Journal 54 (11) (2008) 29432956. 


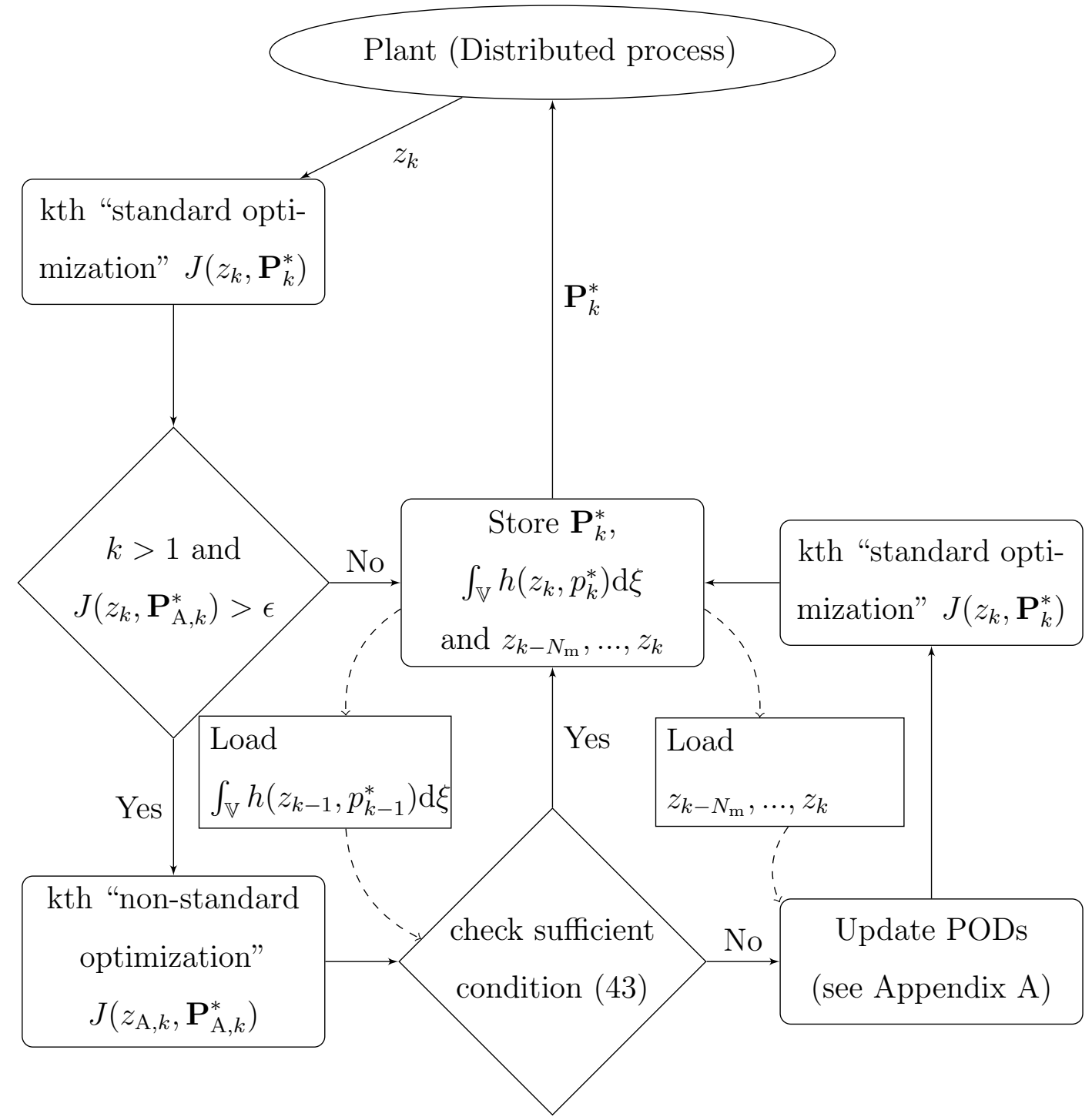

Figure 1: Illustrative flow chart of the MMPC algorithm. 

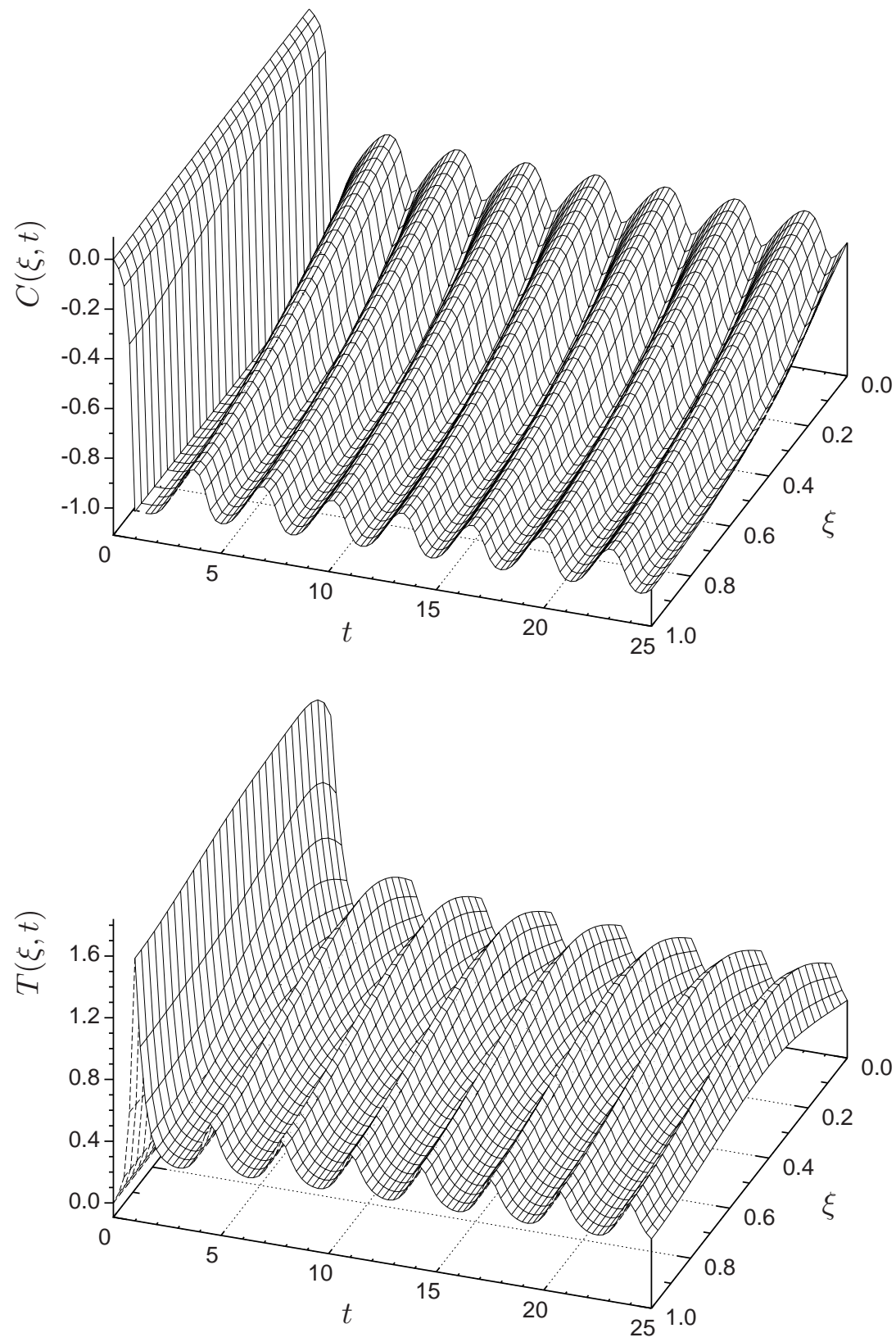

Figure 2: Open-loop concentration and temperature responses. 

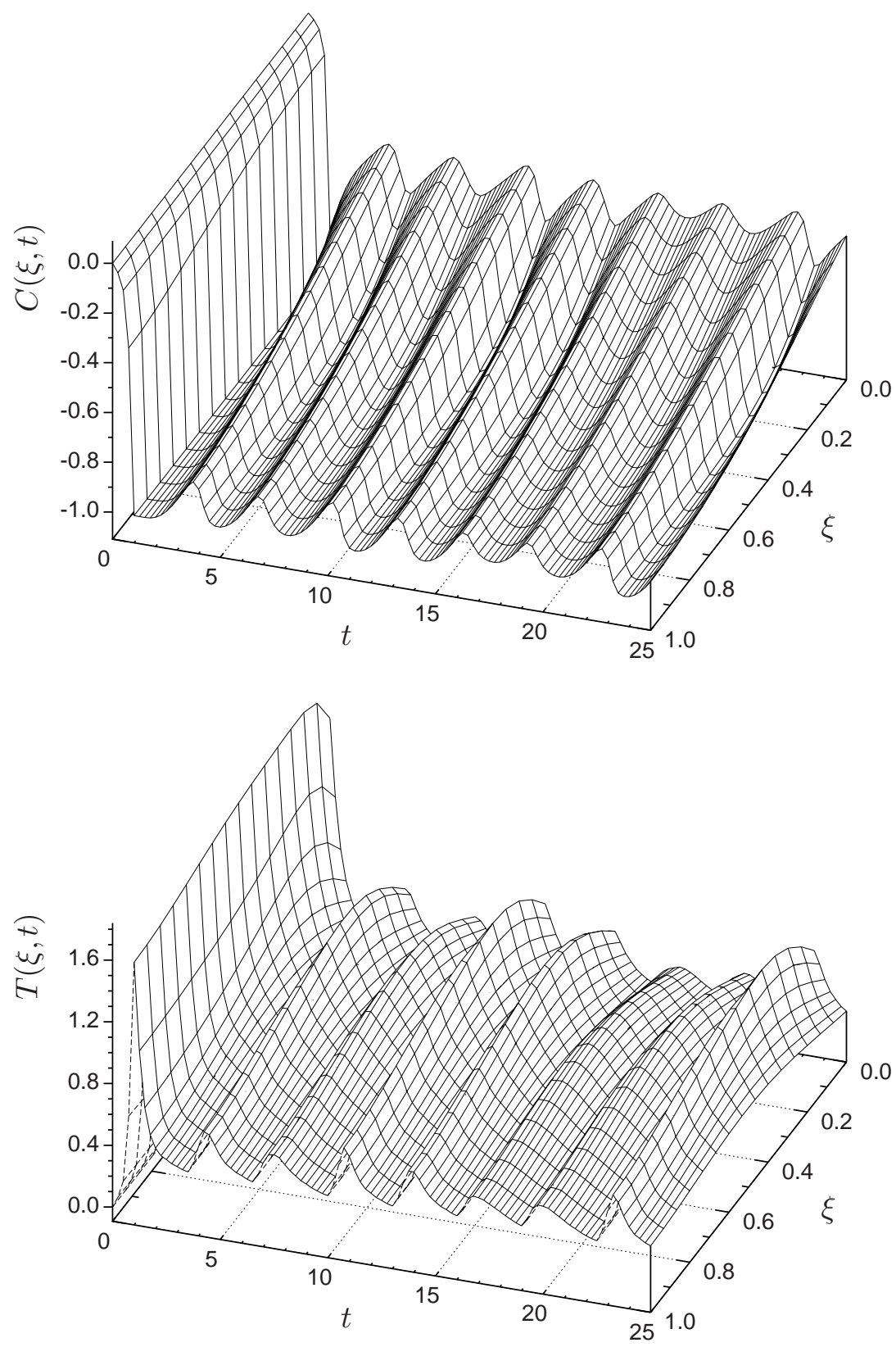

Figure 3: Closed-loop concentration and temperature responses with a conventional MPC strategy using a ROM that captures $99.9 \%$ of the energy. 

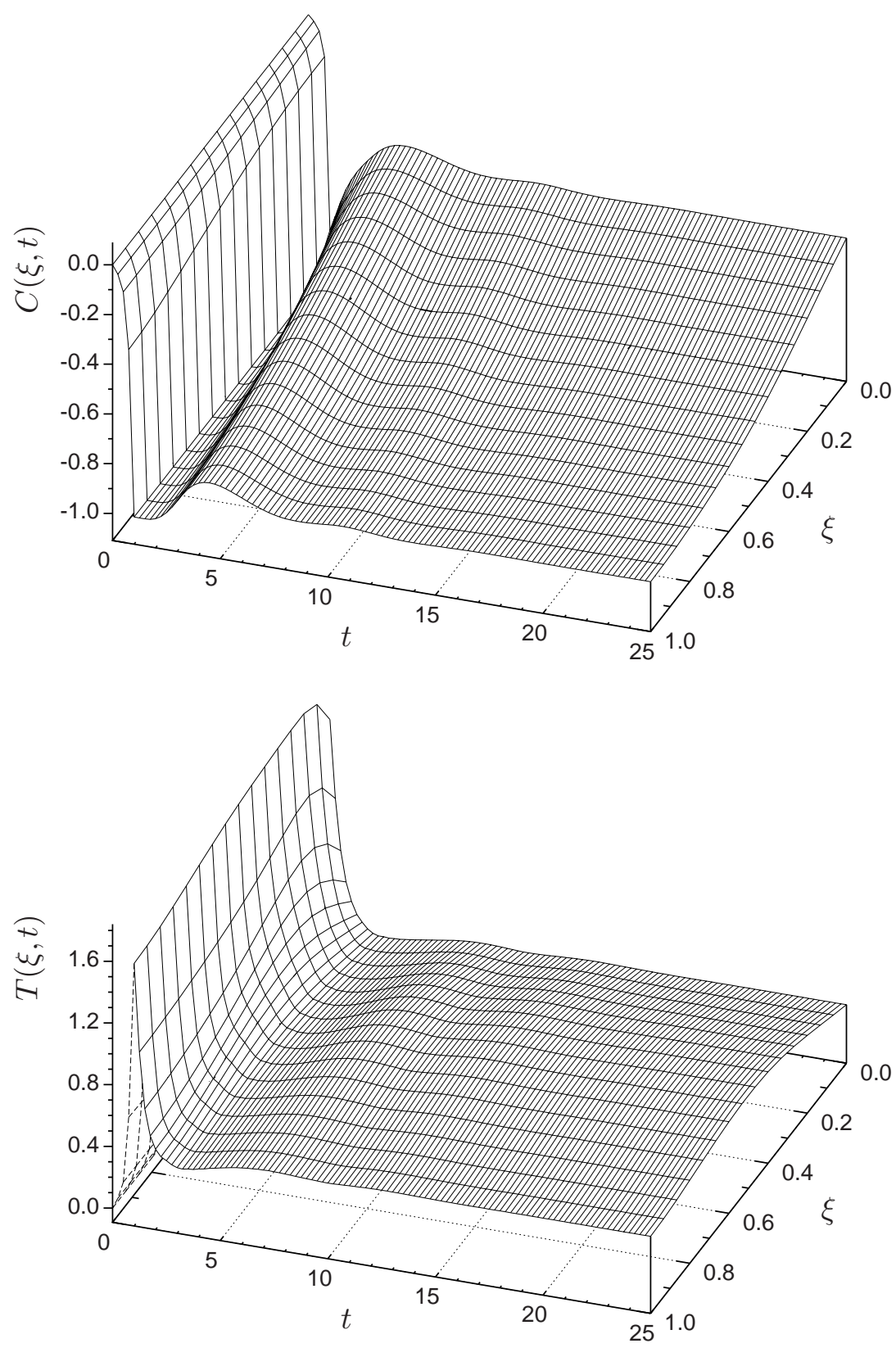

Figure 4: Closed-loop concentration and temperature responses with the MMPC strategy using updated ROMs that capture $99.5 \%$ of the energy. 

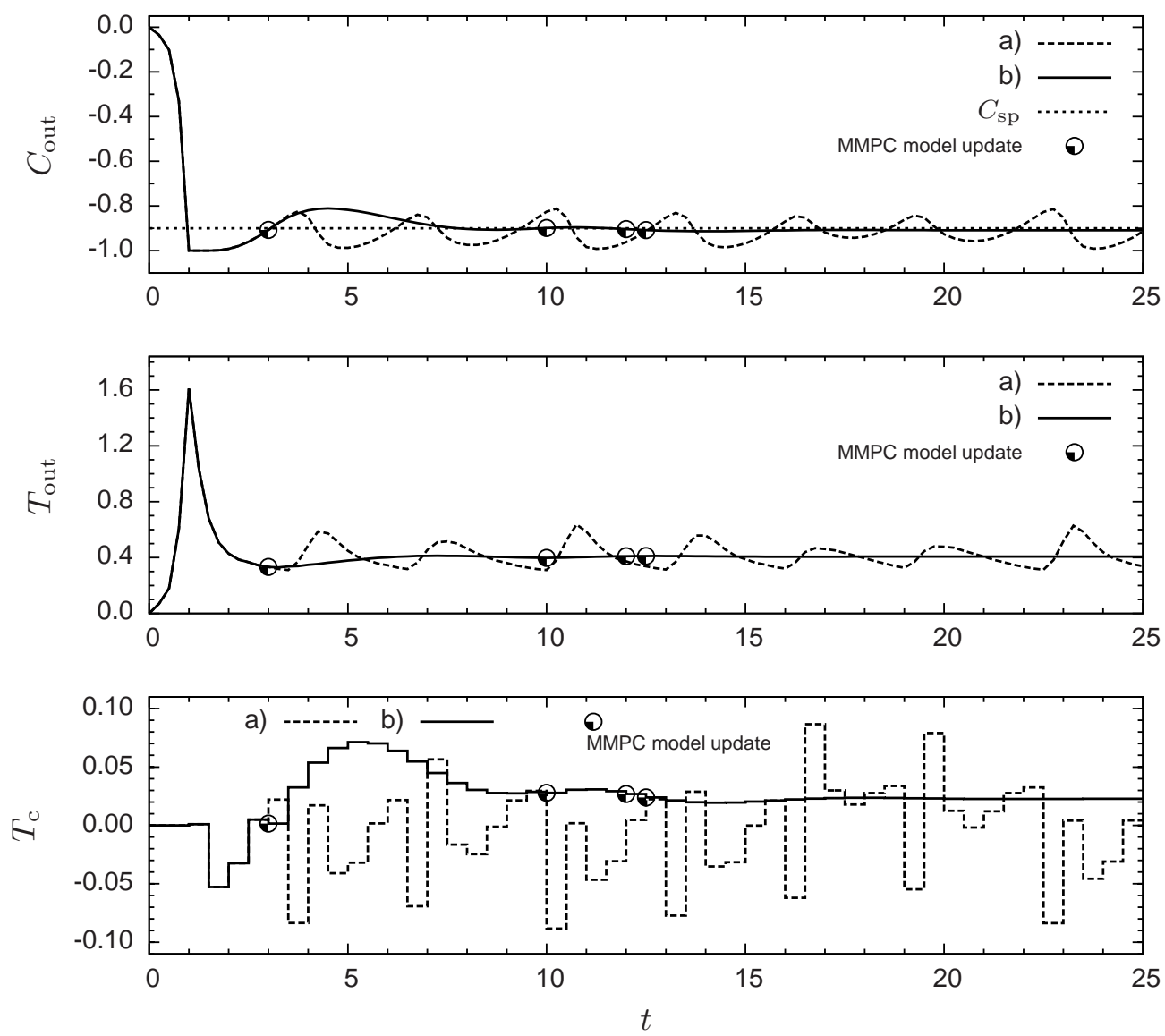

Figure 5: Outlet reactor concentration and temperature closed-loop responses, and controller actuation: a) conventional MPC; b) MMPC strategy. The dots on the MMPC profiles indicate when a model update occurred. 

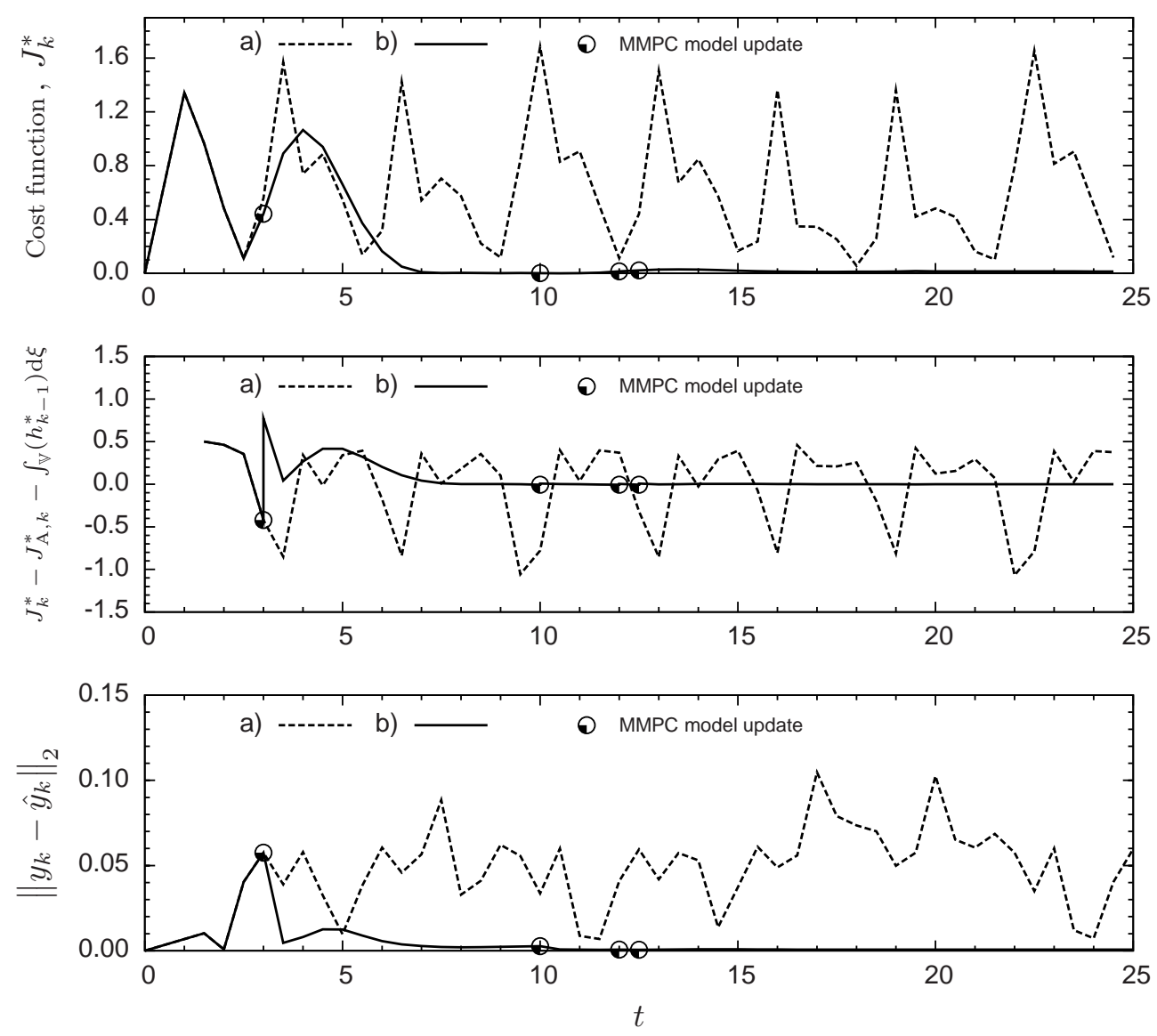

Figure 6: Evolution of the optimal cost function in (46a), inequality (43), and of the states plant/model mismatch: a) conventional MPC; b) MMPC strategy. The dots on the MMPC profiles indicate when a model update occurred. 